\title{
Activation of C-H/H-H Bonds by Rhodium(II) Porphyrin Bimetalloradicals
}

\author{
Weihong Cui and Bradford B. Wayland \\ Department of Chemistry, University of Pennsylvania, Philadelphia, PA 19104-6323
}

\section{Supporting Information}

$\mathbf{N a}\left[{ }^{\mathrm{I}} \mathbf{R h}(\mathbf{m}-\mathbf{x y l y l}) \mathbf{R h}^{\mathrm{I}}\right] \mathbf{N a :}$ The iodorhodium(III) diporphyrin complex I-Rh(m-xylyl)Rh$\mathrm{I}^{1}(43.0 \mathrm{mg}, 0.021 \mathrm{mmol})$ was dissolved in ethanol $(15 \mathrm{~mL})$ by stirring at $60^{\circ} \mathrm{C}$ for $40 \mathrm{~min}$. The resulting deep red solution was filtered in air into a two-neck round-bottom flask and flushed with argon for $40 \mathrm{~min}$. Addition of an excess of $\mathrm{NaBH}_{4}(10.0 \mathrm{mg}, 0.262 \mathrm{mmol})$ in aqueous $\mathrm{NaOH}(0.1$ $\mathrm{M}, 1.4 \mathrm{~mL}$ ) to the above solution under argon effected a color change from deep red to dark brown, indicating the formation of the dianion $\left(\mathrm{Na}\left[{ }^{\mathrm{I}} \mathrm{Rh}(\mathrm{m}-\mathrm{xylyl}) \mathrm{Rh}^{\mathrm{I}}\right] \mathrm{Na}\right)$. The resulting solution was stirred for $1.5 \mathrm{~h}$, and then used in the preparation of organo-rhodium complexes.

$\mathbf{R C H}_{2}-\mathbf{R h}(\mathbf{m}-x y l y l) R h-\mathrm{CH}_{2} \mathbf{R}\left(\mathbf{R}=\mathbf{H}, \mathbf{C H}_{3}\right.$ and $\left.\mathbf{C}_{6} \mathbf{H}_{5}\right)$ : Addition of $\mathrm{RCH}_{2} \mathrm{I}$ (or $\mathrm{RCH}_{2} \mathrm{Br}$ ) to the an ethanol solution of $\mathrm{Na}\left[{ }^{\mathrm{I}} \mathrm{Rh}(\mathrm{m}-\mathrm{xylyl}) \mathrm{Rh}^{\mathrm{I}}\right] \mathrm{Na}$ resulted in the formation of dialkylrhodium(III) diporphyrin complexes as light orange precipitate. The dialkyl derivatives were collected by filtration and purified by column chromatography using silica gel.

H-Rh(m-xylyl)Rh-H: The dihydride complex was prepared using a method similar to that used in the preparation of $\mathrm{CH}_{3}-\mathrm{Rh}\left(\mathrm{m}\right.$-xylyl) $\mathrm{Rh}-\mathrm{CH}_{3}$ except that acetic acid was added to the dianion solution instead of $\mathrm{CH}_{3}$ I. The dihyride complex was filtered under $\mathrm{N}_{2}$ and dried on vacuum line. 
$\mathbf{R}(\mathbf{O H}) \mathbf{C H}-\mathbf{R h}(\mathbf{m}-\mathbf{x y l y l}) \mathbf{R h}-\mathbf{C H}(\mathbf{O H}) \mathbf{R}\left(\mathbf{R}=\mathbf{H}\right.$ or $\left.\mathbf{C H}_{3}\right): 1 \mathrm{mg}$ each of $\mathrm{H}-\mathrm{Rh}(\mathrm{m}-$ xylyl)Rh- $\mathrm{H}$ and $\left(\mathrm{CH}_{2} \mathrm{O}\right)_{x}$ are placed in a vacuum adapted NMR tube, and the reaction solution was prepared by vacuum transfer of $\mathrm{C}_{6} \mathrm{D}_{6}$ as solvent. The sample was pressurized with 300torr of $\mathrm{H}_{2}$, and then heated in the $353 \mathrm{~K}$ water bath for six hours. A single species identified as $\mathrm{CH}_{2} \mathrm{OH}-\mathrm{Rh}(\mathrm{m}-$ xylyl) $\mathrm{Rh}-\mathrm{CH}_{2} \mathrm{OH}$ is observed in the ${ }^{1} \mathrm{H}$ NMR. Vacuum transfer of $\mathrm{CH}_{3} \mathrm{CHO}$ to a benzene solution of $\mathrm{H}-\mathrm{Rh}(\mathrm{m}-\mathrm{xylyl}) \mathrm{Rh}-\mathrm{H}$ in a vacuum adapted NMR tube resulted in formation of a single species identified as $\mathrm{CH}_{3}(\mathrm{OH}) \mathrm{CH}-\mathrm{Rh}(\mathrm{m}-\mathrm{xylyl}) \mathrm{Rh}-\mathrm{CH}(\mathrm{OH}) \mathrm{CH}_{3} .{ }^{1} \mathrm{H}$ NMR $\left(\mathrm{C}_{6} \mathrm{D}_{6}, 296 \mathrm{~K}\right), \delta(\mathrm{ppm}): 8.94(\mathrm{~d}$, $4 \mathrm{H},{ }^{3} J_{\mathrm{H}-\mathrm{H}}=4.9 \mathrm{~Hz}$, pyrrole), $8.79\left(\mathrm{~d}, 4 \mathrm{H},{ }^{3} J_{\mathrm{H}-\mathrm{H}}=4.9 \mathrm{~Hz}\right.$, pyrrole), 8.77 (s, $8 \mathrm{H}$, pyrrole), 8.11 (dd, $2 \mathrm{H}$, ${ }^{4} J_{\mathrm{H}-\mathrm{H}}=2.2 \mathrm{~Hz},{ }^{3} J_{\mathrm{H}-\mathrm{H}}=8.3 \mathrm{~Hz}, o$-phenyl), $7.98\left(\mathrm{dd}, 2 \mathrm{H},{ }^{4} J_{\mathrm{H}-\mathrm{H}}=2.2 \mathrm{~Hz},{ }^{3} J_{\mathrm{H}-\mathrm{H}}=8.3 \mathrm{~Hz}, o^{\prime}-\right.$ phenyl), 7.30 (dd, $2 \mathrm{H},{ }^{4} J_{\mathrm{H}-\mathrm{H}}=2.2 \mathrm{~Hz},{ }^{3} J_{\mathrm{H}-\mathrm{H}}=8.3 \mathrm{~Hz}, m$-phenyl), 7.17 (dd, $2 \mathrm{H},{ }^{4} J_{\mathrm{H}-\mathrm{H}}=2.2 \mathrm{~Hz},{ }^{3} J_{\mathrm{H}-\mathrm{H}}=8.3 \mathrm{~Hz}, m^{\prime}$-phenyl), 7.76 (s. 1H, $o^{\prime \prime}$-phenyl), 7.21 (s. 6H, $m^{\prime \prime \prime}$-phenyl), 7.11 (s. 6H, $m^{\prime \prime \prime \prime}$-phenyl), 7.46 (m, 2H, $o^{\prime \prime \prime}$-phenyl), $7.35\left(\mathrm{~m}, 1 \mathrm{H}, o^{\prime \prime \prime \prime}\right.$-phenyl), $5.00\left(\mathrm{~s}, 4 \mathrm{H},-\mathrm{OCH}_{2}-\right), 2.45\left(\mathrm{~s}, 18 \mathrm{H}, p-\mathrm{CH}_{3}\right), 2.43\left(\mathrm{~s}, 12 \mathrm{H}, o-\mathrm{CH}_{3}\right), 2.17$ ( $\left.\mathrm{s}, 6 \mathrm{H} o^{\prime}-\mathrm{CH}_{3}\right), 2.06\left(\mathrm{~s}, 6 \mathrm{H}, o^{\prime \prime}-\mathrm{CH}_{3}\right), 1.94\left(\mathrm{~s}, 12 \mathrm{H}, o^{\prime \prime \prime}-\mathrm{CH}_{3}\right),-1.56\left(\mathrm{~m}, 2 \mathrm{H},{ }^{3} J_{\mathrm{H}-\mathrm{H}}=4.9 \mathrm{~Hz},{ }^{3} J_{\mathrm{H}-}\right.$ $\left.{ }_{\mathrm{H}}=5.5 \mathrm{~Hz}, \mathrm{Rh}-\mathrm{CH}_{2}(\mathrm{OH}) \mathrm{CH}_{3}\right),-3.41\left(\mathrm{~d} .6 \mathrm{H},{ }^{3} J_{\mathrm{H}-\mathrm{H}}=4.9 \mathrm{~Hz}, \mathrm{Rh}_{-} \mathrm{CH}_{2}(\mathrm{OH}) C_{3}\right),-3.57\left(\mathrm{~d} .6 \mathrm{H},{ }^{3} J_{\mathrm{H}-}\right.$ $\left.{ }_{\mathrm{H}}=4.9 \mathrm{~Hz}, \mathrm{Rh}-\mathrm{CH}_{2}(\mathrm{OH}) \mathrm{CH}_{3}\right)$.

$\cdot \mathbf{R h}(\mathbf{m}-\mathbf{x y l y l}) \mathbf{R h} \cdot(\mathbf{1})$ : Degassed benzene solution of dimethyl dirhodium(III) diporphyrin complex $(\sim 1.0 \mathrm{mg} / 0.3 \mathrm{ml})$ in a vacuum-adapted NMR tube was photolyzed $(\lambda>350 \mathrm{~nm})$ for 6 hours in a Rayonet photoreactor. The solvent along with the methane and toluene formed in the photolysis was removed under vacuum to give $\mathbf{1}$ as a solid in yields of greater than $90 \%$. ${ }^{1} \mathrm{H}$ NMR $\left(\mathrm{C}_{6} \mathrm{D}_{6}, 296 \mathrm{~K}\right), \delta(\mathrm{ppm}): 18.4$ (br s. $16 \mathrm{H}$, pyrrole), 11.3 (br s. $4 \mathrm{H}, o$-phenyl), 9.14(br s. $4 \mathrm{H}, m-$ phenyl), 8.88(br s. $12 \mathrm{H}, m^{\prime}$-phenyl), 8.82 (br s. $1 \mathrm{H}, o^{\prime}$-phenyl), 8.33 (br s. $2 \mathrm{H}, o^{\prime \prime}$-phenyl), 8.06(br s. $1 \mathrm{H}, m^{\prime \prime}$-phenyl), 6.20(br s. $4 \mathrm{H},-\mathrm{OCH}_{2}$-), 3.70(br s. $36 \mathrm{H}, o$-methyl), 3.45(br s. $18 \mathrm{H}, p$-methyl).

$\mathbf{R h}\left(\mathbf{m}\right.$-xylyl)Rh-( $\left.\mu-\mathbf{C H}_{2} \mathbf{C H}_{2}\right): \cdot \operatorname{Rh}(m-x y l y l) R h \cdot(\mathbf{1})$ disappears immediately after the sample solution is pressurized with 460 torr of $\mathrm{CH}_{2}=\mathrm{CH}_{2}$ at room temperature and the ethenebridged complex is confirmed as the only product of the reaction by the ${ }^{1} \mathrm{H} N M R\left(\mathrm{C}_{6} \mathrm{D}_{6}\right) . \delta(\mathrm{ppm})$ $8.51\left(\mathrm{~d}, 2 \mathrm{H},{ }^{3} J_{\mathrm{H}-\mathrm{H}}=5.1 \mathrm{~Hz}\right.$, pyrrole $), 8.47\left(\mathrm{~d}, 2 \mathrm{H},{ }^{3} J_{\mathrm{H}-\mathrm{H}}=4.6 \mathrm{~Hz}\right.$, pyrrole $), 8.41\left(\mathrm{~d}, 2 \mathrm{H},{ }^{3} J_{\mathrm{H}-\mathrm{H}}=4.6 \mathrm{~Hz}\right.$, 
pyrrole), $8.35\left(\mathrm{~d}, 2 \mathrm{H},{ }^{3} J_{\mathrm{H}-\mathrm{H}}=5.1 \mathrm{~Hz}\right.$, pyrrole $), 8.31\left(\mathrm{~d}, 2 \mathrm{H},{ }^{3} J_{\mathrm{H}-\mathrm{H}}=4.6 \mathrm{~Hz}\right.$, pyrrole $), 8.20\left(\mathrm{~d}, 2 \mathrm{H},{ }^{3} J_{\mathrm{H}-\mathrm{H}}\right.$ $=4.6 \mathrm{~Hz}$, pyrrole), $8.06\left(\mathrm{~d}, 2 \mathrm{H},{ }^{3} J_{\mathrm{H}-\mathrm{H}}=4.6 \mathrm{~Hz}\right.$, pyrrole $), 7.99\left(\mathrm{~d}, 2 \mathrm{H},{ }^{3} J_{\mathrm{H}-\mathrm{H}}=4.6 \mathrm{~Hz}\right.$, pyrrole $), 7.64$ (s, 1H,. o-spacer-phenyl), 7.67, 7.65, 7.64, 7.63 (d, $1 \mathrm{H}$ each, ${ }^{4} J_{\mathrm{H}-\mathrm{H}}=1.9 \mathrm{~Hz}$, o-phenyl), 7.24, 7.23, 7.06, 7.05, 7.03, 7.02 (s, 1H each, m-phenyl), 7.26 (m, 1H, m-space phenyl), 6.99, 6.97 (d, 1H each, ${ }^{4} J_{\mathrm{H}-\mathrm{H}}=2.5 \mathrm{~Hz}, \mathrm{o}$-spacer-phenyl) $5.25,5.14$ (each a doublet, $2 \mathrm{H},{ }^{3} J_{\mathrm{H}-\mathrm{H}}=15 \mathrm{~Hz},-\mathrm{OCH}_{\mathrm{a}} \mathrm{H}_{\mathrm{b}}-$ ), 2.47, 2.42, $2.38\left(\mathrm{~S}, 6 \mathrm{H}\right.$ each, $\left.\mathrm{p}-\mathrm{CH}_{3}\right), 2.37,1.77,1.46,1.40,1.26,1.02\left(\mathrm{~S}, 6 \mathrm{H}\right.$ each, o- $\left.\mathrm{CH}_{3}\right),-7.18,-8.38$ (m, $4 \mathrm{H}, \mu-\mathrm{CH}_{A} H_{M} C H_{A} H_{M}$ ).

H-Rh(m-xylyl)Rh-H (2): ${ }^{1} \mathrm{H}$ NMR $\left(\mathrm{C}_{6} \mathrm{D}_{6}\right), \delta(\mathrm{ppm}) 8.94\left(\mathrm{~d}, 4 \mathrm{H},{ }^{3} \mathrm{~J}_{\mathrm{H}-\mathrm{H}}=5.0 \mathrm{~Hz}\right.$, pyrrole), $8.82\left(\mathrm{~d}, 4 \mathrm{H},{ }^{3} J_{\mathrm{H}-\mathrm{H}}=5.0 \mathrm{~Hz}\right.$, pyrrole), $8.81(\mathrm{~s}, 8 \mathrm{H}$, pyrrole $), 8.13\left(\mathrm{dd}, 2 \mathrm{H},{ }^{3} J_{\mathrm{H}-\mathrm{H}}=8.2 \mathrm{~Hz},{ }^{4} J_{\mathrm{H}-\mathrm{H}}=1.9 \mathrm{~Hz}\right.$, o-phenyl), 7.90(dd, $2 \mathrm{H},{ }^{3} J_{\mathrm{H}-\mathrm{H}}=8.2 \mathrm{~Hz},{ }^{4} J_{\mathrm{H}-\mathrm{H}}=1.9 \mathrm{~Hz}, \mathrm{o}$-phenyl), 7.75(s, $1 \mathrm{H}$, o-spacer-phenyl), $7.44\left(\mathrm{~d}, 2 \mathrm{H},{ }^{3} J_{\mathrm{H}-\mathrm{H}}=7.0 \mathrm{~Hz}, \mathrm{o}^{\prime}\right.$-spacer-phenyl), $7.35\left(\mathrm{t}, 1 \mathrm{H} .{ }^{3} J_{\mathrm{H}-\mathrm{H}}={ }^{3} J_{\mathrm{H}-\mathrm{H}}=7.0 \mathrm{~Hz}, \mathrm{~m}\right.$-spacerphenyl), $7.32\left(\mathrm{dd}, 2 \mathrm{H},{ }^{3} J_{\mathrm{H}-\mathrm{H}}=8.3 \mathrm{~Hz},{ }^{4} J_{\mathrm{H}-\mathrm{H}}=2.5 \mathrm{~Hz}, \mathrm{~m}\right.$-phenyl), $7.11\left(\mathrm{dd}, 2 \mathrm{H},{ }^{3} J_{\mathrm{H}-\mathrm{H}}=8.3 \mathrm{~Hz},{ }^{4} J_{\mathrm{H}-\mathrm{H}}=\right.$ $2.5 \mathrm{~Hz}, \mathrm{~m}$--phenyl), 7.22(s, 6H, m"-phenyl), 7.20(s, 2H, m"'-phenyl), 7.11(s, 2H, m"'--phenyl), 7.10(s, 6H, m"'"-phenyl), 4.98(s, 4H, - $\left.\mathrm{OCH}_{2}-\right), 2.43\left(\mathrm{~s}, 18 \mathrm{H}, \mathrm{p}-\mathrm{CH}_{3}\right), 2.28\left(\mathrm{~s}, 12 \mathrm{H}, \mathrm{o}-\mathrm{CH}_{3}\right), 2.05(\mathrm{~s}$, $\left.6 \mathrm{H}, \mathrm{o}^{\prime}-\mathrm{CH}_{3}\right), 1.87\left(\mathrm{~s}, 6 \mathrm{H}, \mathrm{o}^{\prime \prime}-\mathrm{CH}_{3}\right), 1.76\left(\mathrm{~s}, 12 \mathrm{H}, \mathrm{o}^{\prime \prime}-\mathrm{CH}_{3}\right),-40.06\left(\mathrm{~d}, 2 \mathrm{H}, \mathrm{Rh}-\mathrm{H}_{3},{ }^{1} J_{103_{\mathrm{Rh}-\mathrm{H}}}=42.5 \mathrm{~Hz}\right)$.

${ }^{\mathrm{a}} \mathbf{R h}(\mathbf{m}-\mathbf{x y l y l}) \mathbf{R h}^{\mathrm{b}}-\mathbf{H}(\mathbf{3}):{ }^{1} \mathrm{H}$ NMR $\left(\mathrm{C}_{6} \mathrm{D}_{6}, 296 \mathrm{~K}\right), \delta(\mathrm{ppm}): 5.90\left(\mathrm{~s}, 2 \mathrm{H},-\mathrm{OCH}_{2^{-}}{ }^{\mathrm{a}}\right), 5.31(\mathrm{~s}$, $\left.2 \mathrm{H},-\mathrm{OCH}_{2}{ }^{-}{ }^{\mathrm{b}}\right),-40.03\left(\mathrm{~d}, 1 \mathrm{H},{ }^{1} J_{103_{\mathrm{Rh}-\mathrm{H}}}=42.5 \mathrm{~Hz}, \mathrm{Rh}-H\right)$.

$\mathbf{C H}_{3}-\mathbf{R h}(\mathbf{m}-\mathbf{x y l y l}) \mathbf{R h}-\mathbf{C H}_{3}$ (4): ${ }^{1} \mathrm{H}$ NMR $\left(\mathrm{C}_{6} \mathrm{D}_{6}\right), \delta(\mathrm{ppm}) 8.97\left(\mathrm{~d}, 4 \mathrm{H},{ }^{3} J_{\mathrm{H}-\mathrm{H}}=4.9 \mathrm{~Hz}\right.$, pyrrole), $8.81\left(\mathrm{~d}, 4 \mathrm{H},{ }^{3} J_{\mathrm{H}-\mathrm{H}}=4.9 \mathrm{~Hz}\right.$, pyrrole), $8.77\left(\mathrm{~s}, 8 \mathrm{H}\right.$, pyrrole), $8.16\left(\mathrm{dd}, 2 \mathrm{H},{ }^{3} J_{\mathrm{H}-\mathrm{H}}=8.3 \mathrm{~Hz},{ }^{4} J_{\mathrm{H}-\mathrm{H}}\right.$ $=2.2 \mathrm{~Hz}, \mathrm{o}$-phenyl), $8.03\left(\mathrm{dd}, 2 \mathrm{H},{ }^{3} J_{\mathrm{H}-\mathrm{H}}=8.3 \mathrm{~Hz},{ }^{4} J_{\mathrm{H}-\mathrm{H}}=2.2 \mathrm{~Hz}, \mathrm{o}\right.$-phenyl), $7.76(\mathrm{~s}, 1 \mathrm{H},$. o-spacerphenyl), 7.46(d, $2 \mathrm{H},{ }^{3} J_{\mathrm{H}-\mathrm{H}}=7.0 \mathrm{~Hz}, \mathrm{o}^{\prime}$-spacer-phenyl), $7.36\left(\mathrm{t}, 1 \mathrm{H} .{ }^{3} J_{\mathrm{H}-\mathrm{H}}=7.0 \mathrm{~Hz}\right.$, m-spacer-phenyl), $7.33\left(\mathrm{dd}, 2 \mathrm{H},{ }^{3} J_{\mathrm{H}-\mathrm{H}}=8.3 \mathrm{~Hz},{ }^{4} J_{\mathrm{H}-\mathrm{H}}=2.6 \mathrm{~Hz}, \mathrm{~m}-\right.$ phenyl), $7.17\left(\mathrm{dd}, 2 \mathrm{H},{ }^{3} J_{\mathrm{H}-\mathrm{H}}=8.3 \mathrm{~Hz},{ }^{4} J_{\mathrm{H}-\mathrm{H}}=2.6 \mathrm{~Hz}\right.$, m'-phenyl), 7.24(s, 6H, m"-phenyl), 7.20(s, 2H, m"'-phenyl), 7.10(s, 2H, m"'--phenyl), 7.08(s, 6H, m"'"'-phenyl), 5.00(s, 4H, - $\left.\mathrm{OCH}_{2}-\right), 2.45\left(\mathrm{~s}, 18 \mathrm{H}, \mathrm{p}-\mathrm{CH}_{3}\right), 2.33\left(\mathrm{~s}, 12 \mathrm{H}, \mathrm{o}-\mathrm{CH}_{3}\right), 2.22\left(\mathrm{~s}, 6 \mathrm{H}, \mathrm{o}^{\prime}-\mathrm{CH}_{3}\right)$, $1.77\left(\mathrm{~s}, 6 \mathrm{H}, \mathrm{o}\right.$ "- $\left.\mathrm{CH}_{3}\right), 1.72\left(\mathrm{~s}, 12 \mathrm{H}, \mathrm{o}\right.$ "'- $\left.\mathrm{CH}_{3}\right),-5.29\left(\mathrm{~d}, 6 \mathrm{H}, \mathrm{Rh}_{-} \mathrm{CH}_{3},{ }^{2} J_{103_{\mathrm{Rh}-\mathrm{H}}}=2.9 \mathrm{~Hz}\right)$. 
${ }^{\mathrm{a}} \mathbf{R h}(\mathbf{m}-\mathbf{x y l y l}) \mathbf{R h}^{\mathrm{b}}-\mathrm{CH}_{3}(\mathbf{5}):{ }^{1} \mathrm{H}$ NMR $\left(\mathrm{C}_{6} \mathrm{D}_{6}, 296 \mathrm{~K}\right), \delta(\mathrm{ppm}): 5.90\left(\mathrm{~s}, 2 \mathrm{H},-\mathrm{OCH}_{2^{-}}{ }^{\mathrm{a}}\right)$, $5.31\left(\mathrm{~s}, 2 \mathrm{H},-\mathrm{OCH}_{2}{ }^{-}{ }^{\mathrm{b}}\right),-5.27\left(\mathrm{~d}, 6 \mathrm{H}, \mathrm{Rh}_{-} \mathrm{CH}_{3},{ }^{2} J_{103 \mathrm{Rh}-\mathrm{H}}=2.9 \mathrm{~Hz}\right)$.

H-Rha $(\mathbf{m}-\mathbf{x y l y l}) \mathbf{R h}^{\mathrm{b}}-\mathbf{C H}_{3}(\mathbf{6}):{ }^{1} \mathrm{H}$ NMR $\left(\mathrm{C}_{6} \mathrm{D}_{6}, 296 \mathrm{~K}\right), \delta(\mathrm{ppm}): 8.95\left(\mathrm{~d}, 2 \mathrm{H},{ }^{3} J_{\mathrm{H}-\mathrm{H}}=4.9 \mathrm{~Hz}\right.$, pyrrole $), 8.97\left(\mathrm{~d}, 2 \mathrm{H},{ }^{3} J_{\mathrm{H}-\mathrm{H}}=4.9 \mathrm{~Hz}\right.$, pyrrole $\left.^{\mathrm{b}}\right), 8.82\left(\mathrm{~d}, 2 \mathrm{H},{ }^{3} J_{\mathrm{H}-\mathrm{H}}=4.9 \mathrm{~Hz}\right.$, pyrrole $), 8.81\left(\mathrm{~d}, 2 \mathrm{H},{ }^{3} J_{\mathrm{H}-}\right.$ ${ }_{\mathrm{H}}=4.9 \mathrm{~Hz}$, pyrrole $\left.^{\mathrm{b}}\right), 8.80(\mathrm{~s}, 4 \mathrm{H}$, pyrrole $), 8.78\left(\mathrm{~s}, 4 \mathrm{H}\right.$, pyrrole $\left.^{\mathrm{b}}\right), 8.13\left(\mathrm{dd}, 1 \mathrm{H},{ }^{4} J_{\mathrm{H}-\mathrm{H}}=2 \mathrm{~Hz},{ }^{3} J_{\mathrm{H}-}\right.$ ${ }_{\mathrm{H}}=8.3 \mathrm{~Hz}, o$-phenyl $\left.{ }^{\mathrm{a}}\right), 8.16\left(\mathrm{dd}, 1 \mathrm{H},{ }^{4} J_{\mathrm{H}-\mathrm{H}}=2 \mathrm{~Hz},{ }^{3} J_{\mathrm{H}-\mathrm{H}}=8.3 \mathrm{~Hz}, o\right.$-phenyl $\left.{ }^{\mathrm{b}}\right), 7.90\left(\mathrm{dd}, 1 \mathrm{H},{ }^{4} J_{\mathrm{H}-\mathrm{H}}=2.2 \mathrm{~Hz}\right.$, ${ }^{3} J_{\mathrm{H}-\mathrm{H}}=8.3 \mathrm{~Hz}, o^{\prime}-$ phenyl $\left.{ }^{\mathrm{a}}\right), 8.03\left(\mathrm{dd}, 1 \mathrm{H},{ }^{4} J_{\mathrm{H}-\mathrm{H}}=2.2 \mathrm{~Hz},{ }^{3} J_{\mathrm{H}-\mathrm{H}}=8.3 \mathrm{~Hz}, o^{\prime}-\right.$ phenyl $\left.{ }^{\mathrm{b}}\right), 7.31\left(\mathrm{dd}, 1 \mathrm{H},{ }^{4} J_{\mathrm{H}-}\right.$ ${ }_{\mathrm{H}}=2.2 \mathrm{~Hz},{ }^{3} J_{\mathrm{H}-\mathrm{H}}=8.3 \mathrm{~Hz}, m$-phenyl $\left.{ }^{\mathrm{a}}\right), 7.33\left(\mathrm{dd}, 1 \mathrm{H},{ }^{4} J_{\mathrm{H}-\mathrm{H}}=2.2 \mathrm{~Hz},{ }^{3} J_{\mathrm{H}-\mathrm{H}}=8.3 \mathrm{~Hz}, m\right.$-phenyl $\left.{ }^{\mathrm{b}}\right), 7.17(\mathrm{dd}$, $2 \mathrm{H},{ }^{4} J_{\mathrm{H}-\mathrm{H}}=2.2 \mathrm{~Hz},{ }^{3} J_{\mathrm{H}-\mathrm{H}}=8.3 \mathrm{~Hz}, m^{\prime}$-phenyl $\left.{ }^{\mathrm{a}, \mathrm{b}}\right), 7.76$ (s. $1 \mathrm{H}, o$-spacer-phenyl), 7.44 (m, $2 \mathrm{H}, o^{\prime}$-spacerphenyl), 7.36 (m, 1H, $m$-spacer-phenyl), 7.22 (s. 3H, $m^{\prime \prime}$-phenyla), 7.23 (s. 3H, $m^{\prime \prime}$-phenyl ${ }^{\mathrm{b}}$ ), 7.10 (s. 3H, $m^{\prime \prime \prime}$-phenyl $\left.1^{\mathrm{a}}\right), 7.07$ (s. 3H, $m^{\prime \prime \prime}$-phenyl $\left.{ }^{\mathrm{b}}\right), 4.99\left(\mathrm{~s}, 4 \mathrm{H},-\mathrm{OCH}_{2^{-}}{ }^{\mathrm{a}, \mathrm{b}}\right), 2.43\left(\mathrm{~s}, 9 \mathrm{H}, p-\mathrm{CH}_{3}{ }^{\mathrm{a}}\right), 2.44$ $\left(\mathrm{s}, 9 \mathrm{H}, p-\mathrm{CH}_{3}{ }^{\mathrm{b}}\right), 2.29\left(\mathrm{~s}, 6 \mathrm{H}, o-\mathrm{CH}_{3}{ }^{\mathrm{a}}\right), 2.33\left(\mathrm{~s}, 6 \mathrm{H}, o-\mathrm{CH}_{3}{ }^{\mathrm{b}}\right), 2.05\left(\mathrm{~s}, 3 \mathrm{H}, o^{\prime}-\mathrm{CH}_{3}{ }^{\mathrm{a}}\right), 2.22\left(\mathrm{~s}, 3 \mathrm{H} o^{\prime}-\right.$ $\left.\mathrm{CH}_{3}{ }^{\mathrm{b}}\right), 1.87\left(\mathrm{~s}, 3 \mathrm{H}, o^{\prime \prime}-\mathrm{CH}_{3}{ }^{\mathrm{a}}\right), 1.77\left(\mathrm{~s}, 3 \mathrm{H}, o^{\prime \prime}-\mathrm{CH}_{3}{ }^{\mathrm{b}}\right), 1.76\left(\mathrm{~s}, 6 \mathrm{H}, o^{\prime \prime \prime}-\mathrm{CH}_{3}{ }^{\mathrm{a}}\right), 1.72\left(\mathrm{~s}, 6 \mathrm{H}, o^{\prime \prime \prime}-\mathrm{CH}_{3}{ }^{\mathrm{b}}\right),-$ $5.29\left(\mathrm{~d}, 3 \mathrm{H},{ }^{2} J_{103_{\mathrm{Rh}-\mathrm{H}}}=2.7 \mathrm{~Hz}\right),-40.06\left(\mathrm{~d}, 1 \mathrm{H},{ }^{1} J_{103_{\mathrm{Rh}} \mathrm{H}}=42.5 \mathrm{~Hz}, \mathrm{Rh}-H\right)$.

$\mathbf{H}-\mathbf{R h}{ }^{\mathrm{a}}(\mathbf{m}-\mathbf{x y l y l}) \mathbf{R h}^{\mathrm{b}}-\mathbf{C H}_{2} \mathbf{C H}_{3}(\mathbf{7}):{ }^{1} \mathrm{H}$ NMR $\left(\mathrm{C}_{6} \mathrm{D}_{6}, 296 \mathrm{~K}\right), \delta(\mathrm{ppm}): 8.95\left(\mathrm{~d}, 2 \mathrm{H},{ }^{3} \boldsymbol{J}_{\mathrm{H}-}\right.$ ${ }_{\mathrm{H}}=4.9 \mathrm{~Hz}$, pyrrole $), 9.07\left(\mathrm{~d}, 2 \mathrm{H},{ }^{3} J_{\mathrm{H}-\mathrm{H}}=4.9 \mathrm{~Hz}\right.$, pyrrole $\left.{ }^{\mathrm{b}}\right), 8.82\left(\mathrm{~d}, 2 \mathrm{H},{ }^{3} J_{\mathrm{H}-\mathrm{H}}=4.9 \mathrm{~Hz}\right.$, pyrrole $\left.{ }^{\mathrm{a}}\right), 8.86(\mathrm{~d}$, $2 \mathrm{H},{ }^{3} J_{\mathrm{H}-\mathrm{H}}=4.9 \mathrm{~Hz}$, pyrrole $\left.{ }^{\mathrm{b}}\right), 8.80(\mathrm{~s}, 4 \mathrm{H}$, pyrrole $), 8.76\left(\mathrm{~s}, 4 \mathrm{H}\right.$, pyrrole $\left.{ }^{\mathrm{b}}\right), 8.13\left(\mathrm{dd}, 1 \mathrm{H},{ }^{4} \mathrm{~J}_{\mathrm{H}-\mathrm{H}}=2 \mathrm{~Hz}\right.$, ${ }^{3} J_{\mathrm{H}-\mathrm{H}}=8.3 \mathrm{~Hz}, o$-phenyl $\left.\mathrm{l}^{\mathrm{a}}\right), 8.16\left(\mathrm{dd}, 1 \mathrm{H},{ }^{4} J_{\mathrm{H}-\mathrm{H}}=2 \mathrm{~Hz},{ }^{3} J_{\mathrm{H}-\mathrm{H}}=8.3 \mathrm{~Hz}, o-\right.$ phenyl $\left.{ }^{\mathrm{b}}\right), 7.90\left(\mathrm{dd}, 1 \mathrm{H},{ }^{4} J_{\mathrm{H}-}\right.$ ${ }_{\mathrm{H}}=2.2 \mathrm{~Hz},{ }^{3} J_{\mathrm{H}-\mathrm{H}}=8.3 \mathrm{~Hz}, o^{\prime}$-phenyl $\left.{ }^{\mathrm{a}}\right), 8.03\left(\mathrm{dd}, 1 \mathrm{H},{ }^{4} J_{\mathrm{H}-\mathrm{H}}=2.2 \mathrm{~Hz},{ }^{3} J_{\mathrm{H}-\mathrm{H}}=8.3 \mathrm{~Hz}, o^{\prime}\right.$-phenyl $\left.\mathrm{b}^{\mathrm{b}}\right), 7.31(\mathrm{dd}$, $1 \mathrm{H},{ }^{4} J_{\mathrm{H}-\mathrm{H}}=2.2 \mathrm{~Hz},{ }^{3} J_{\mathrm{H}-\mathrm{H}}=8.3 \mathrm{~Hz}, m$-phenyl $\left.{ }^{\mathrm{a}}\right), 7.33\left(\mathrm{dd}, 1 \mathrm{H},{ }^{4} J_{\mathrm{H}-\mathrm{H}}=2.2 \mathrm{~Hz},{ }^{3} J_{\mathrm{H}-\mathrm{H}}=8.3 \mathrm{~Hz}, m-\right.$ phenyl $\left.{ }^{\mathrm{b}}\right), 7.17$ $\left(\mathrm{dd}, 2 \mathrm{H},{ }^{4} J_{\mathrm{H}-\mathrm{H}}=2.2 \mathrm{~Hz},{ }^{3} J_{\mathrm{H}-\mathrm{H}}=8.3 \mathrm{~Hz}, m^{\prime}\right.$-phenyl $\left.{ }^{\mathrm{a}, \mathrm{b}}\right), 7.76$ (s. $1 \mathrm{H}, o$-spacer-phenyl), $7.45\left(\mathrm{~m}, 2 \mathrm{H}, o^{\prime}-\right.$ spacer-phenyl), 7.36 (m, 1H, $m$-spacer-phenyl), 7.22 (s. 3H, $m^{\prime \prime}$-phenyl $\left.{ }^{\mathrm{a}}\right), 7.24$ (s. $3 \mathrm{H}, m^{\prime \prime}$-phenyl ${ }^{\mathrm{b}}$ ), 7.10 (s. 3H, $m^{\prime \prime \prime}$-phenyl $\mathrm{l}^{\mathrm{a}}$ ), 7.08 (s. 3H, $m^{\prime \prime \prime}$-phenyl $\left.{ }^{\mathrm{b}}\right), 5.00\left(\mathrm{~s}, 4 \mathrm{H},-\mathrm{OCH}_{2^{-}}{ }^{\mathrm{a}, \mathrm{b}}\right), 2.43\left(\mathrm{~s}, 9 \mathrm{H}, p-\mathrm{CH}_{3}{ }^{\mathrm{a}}\right)$, $2.44\left(\mathrm{~s}, 9 \mathrm{H}, p-\mathrm{CH}_{3}{ }^{\mathrm{b}}\right), 2.29\left(\mathrm{~s}, 6 \mathrm{H}, o-\mathrm{CH}_{3}{ }^{\mathrm{a}}\right), 2.33\left(\mathrm{~s}, 6 \mathrm{H}, o-\mathrm{CH}_{3}{ }^{\mathrm{b}}\right), 2.05\left(\mathrm{~s}, 3 \mathrm{H}, o^{\prime}-\mathrm{CH}_{3}{ }^{\mathrm{a}}\right), 2.24(\mathrm{~s}, 3 \mathrm{H}$ $\left.o^{\prime}-\mathrm{CH}_{3}{ }^{\mathrm{b}}\right), 1.87\left(\mathrm{~s}, 3 \mathrm{H}, o^{\prime \prime}-\mathrm{CH}_{3}{ }^{\mathrm{a}}\right), 1.78\left(\mathrm{~s}, 3 \mathrm{H}, o^{\prime \prime}-\mathrm{CH}_{3}{ }^{\mathrm{b}}\right), 1.72\left(\mathrm{~s}, 12 \mathrm{H}, o^{\prime \prime \prime}-\mathrm{CH}_{3}^{\mathrm{a}, \mathrm{b}}\right),-3.89(\mathrm{dt}, 3 \mathrm{H}, \mathrm{Rh}-$ 
$\left.\mathrm{CH}_{2} \mathrm{CH}_{3},{ }^{2} J_{103_{\mathrm{Rh}-\mathrm{H}}}=1.4 \mathrm{~Hz},{ }^{3} J_{\mathrm{H}-\mathrm{H}}=7.7 \mathrm{~Hz}\right),-4.33\left(\mathrm{dq}, 2 \mathrm{H}, \mathrm{Rh}-\mathrm{CH}_{2} \mathrm{CH}_{3},{ }^{2} J_{103_{\mathrm{Rh}} \mathrm{H}}=2.8 \mathrm{~Hz},{ }^{3} J_{\mathrm{H}-\mathrm{H}}=\right.$ 7.7Hz), $-40.06\left(\mathrm{~d}, 1 \mathrm{H},{ }^{1} J_{103_{\mathrm{Rh}-\mathrm{H}}}=42.5 \mathrm{~Hz}, \mathrm{Rh}-H\right)$.

${ }^{\mathrm{a}} \mathbf{R h}(\mathbf{m}-\mathbf{x y l y l}) \mathbf{R h}^{\mathrm{b}}-\mathbf{C H}_{2} \mathbf{C H}_{3}(\mathbf{8}):{ }^{1} \mathrm{H}$ NMR $\left(\mathrm{C}_{6} \mathrm{D}_{6}, 296 \mathrm{~K}\right), \delta(\mathrm{ppm}): 5.90\left(\mathrm{~s}, 2 \mathrm{H},-\mathrm{OCH}_{2}{ }^{-}{ }^{\mathrm{a}}\right)$, $5.31\left(\mathrm{~s}, 2 \mathrm{H},-\mathrm{OCH}_{2}{ }^{-}{ }^{\mathrm{b}}\right),-3.87\left(\mathrm{~m}, 3 \mathrm{H}, \mathrm{Rh}-\mathrm{CH}_{2} \mathrm{CH}_{3},\right),-4.32\left(\mathrm{~m}, 2 \mathrm{H}, \mathrm{Rh}_{-} \mathrm{CH}_{2} \mathrm{CH}_{3},{ }^{2} J_{103_{\mathrm{Rh}-\mathrm{H}}}=2.8 \mathrm{~Hz},{ }^{3} J_{\mathrm{H}-}\right.$ $\left.{ }_{\mathrm{H}}=7.7 \mathrm{~Hz}\right)$.

$\mathbf{C H}_{3} \mathbf{C H}_{2}-\mathbf{R h}(\mathbf{m}-\mathbf{x y l y l}) \mathbf{R h}-\mathbf{C H}_{2} \mathbf{C H}_{3}$ (9): ${ }^{1} \mathrm{H}$ NMR $\left(\mathrm{C}_{6} \mathrm{D}_{6}\right), \delta(\mathrm{ppm}) 9.07\left(\mathrm{~d}, 4 \mathrm{H},{ }^{3} J_{\mathrm{H}-\mathrm{H}}=4.9\right.$ $\mathrm{Hz}$, pyrrole), $8.86\left(\mathrm{~d}, 4 \mathrm{H},{ }^{3} J_{\mathrm{H}-\mathrm{H}}=4.9 \mathrm{~Hz}\right.$, pyrrole $), 8.76(\mathrm{~s}, 8 \mathrm{H}$, pyrrole $), 8.16\left(\mathrm{dd}, 2 \mathrm{H},{ }^{3} J_{\mathrm{H}-\mathrm{H}}=8.3 \mathrm{~Hz}\right.$, ${ }^{4} J_{\mathrm{H}-\mathrm{H}}=2.2 \mathrm{~Hz}, \mathrm{o}-$ phenyl), $8.03\left(\mathrm{dd}, 2 \mathrm{H},{ }^{3} J_{\mathrm{H}-\mathrm{H}}=8.3 \mathrm{~Hz},{ }^{4} J_{\mathrm{H}-\mathrm{H}}=2.2 \mathrm{~Hz}, \mathrm{o}-\right.$-phenyl), $7.76(\mathrm{~s}, 1 \mathrm{H}, . \mathrm{o}-$ spacer-phenyl), 7.46(d, 2H, ${ }^{3} J_{\mathrm{H}-\mathrm{H}}=7.0 \mathrm{~Hz}, \mathrm{o}^{\prime}$-spacer-phenyl), 7.36(t, $1 \mathrm{H} .{ }^{3} J_{\mathrm{H}-\mathrm{H}}=7.0 \mathrm{~Hz}, \mathrm{~m}$-spacerphenyl), $7.33\left(\mathrm{dd}, 2 \mathrm{H},{ }^{3} J_{\mathrm{H}-\mathrm{H}}=8.3 \mathrm{~Hz},{ }^{4} J_{\mathrm{H}-\mathrm{H}}=2.6 \mathrm{~Hz}, \mathrm{~m}\right.$-phenyl), $7.17\left(\mathrm{dd}, 2 \mathrm{H},{ }^{3} J_{\mathrm{H}-\mathrm{H}}=8.3 \mathrm{~Hz},{ }^{4} J_{\mathrm{H}-\mathrm{H}}=\right.$ $2.6 \mathrm{~Hz}, \mathrm{~m}$ '-phenyl), 7.24(s, 6H, m"-phenyl), 7.20(s, 2H, m"'-phenyl), 7.10(s, 2H, m"'--phenyl), 7.08(s, 6H, m"'"'-phenyl), 5.00(s, 4H, - $\left.\mathrm{OCH}_{2}-\right), 2.44\left(\mathrm{~s}, 18 \mathrm{H}, \mathrm{p}-\mathrm{CH}_{3}\right), 2.33\left(\mathrm{~s}, 12 \mathrm{H}, \mathrm{o}-\mathrm{CH}_{3}\right), 2.24(\mathrm{~s}$, $\left.6 \mathrm{H}, \mathrm{o}^{\prime}-\mathrm{CH}_{3}\right), 1.78\left(\mathrm{~s}, 6 \mathrm{H}, \mathrm{o}\right.$ "- $\left.\mathrm{CH}_{3}\right), 1.72\left(\mathrm{~s}, 12 \mathrm{H}, \mathrm{o}^{\prime \prime \prime}-\mathrm{CH}_{3}\right),-3.89\left(\mathrm{dt}, 6 \mathrm{H}, \mathrm{Rh}_{-} \mathrm{CH}_{2} \mathrm{CH}_{3},{ }^{2} J_{103_{\mathrm{Rh}} \mathrm{H}}=\right.$ $\left.1.4 \mathrm{~Hz},{ }^{3} J_{\mathrm{H}-\mathrm{H}}=7.7 \mathrm{~Hz}\right),-4.33\left(\mathrm{dq}, 4 \mathrm{H}, \mathrm{Rh}-\mathrm{CH}_{2} \mathrm{CH}_{3},{ }^{2} J_{103 \mathrm{Rh}-\mathrm{H}}=2.8 \mathrm{~Hz},{ }^{3} J_{\mathrm{H}-\mathrm{H}}=7.7 \mathrm{~Hz}\right)$.

$\mathbf{H}-\mathbf{R h}{ }^{\mathrm{a}}(\mathbf{m}-\mathbf{x y l y l}) \mathbf{R h}^{\mathrm{b}}-\mathbf{C H}_{2} \mathbf{O H}(\mathbf{1 0}):{ }^{1} \mathrm{H}$ NMR $\left(\mathrm{C}_{6} \mathrm{D}_{6}, 296 \mathrm{~K}\right), \delta(\mathrm{ppm}): 8.95\left(\mathrm{~d}, 2 \mathrm{H},{ }^{3} \mathrm{~J}_{\mathrm{H}-}\right.$ ${ }_{\mathrm{H}}=4.9 \mathrm{~Hz}$, pyrrole $\left.)^{\mathrm{a}}\right), 8.97\left(\mathrm{~d}, 2 \mathrm{H},{ }^{3} J_{\mathrm{H}-\mathrm{H}}=4.9 \mathrm{~Hz}\right.$, pyrrole $\left.{ }^{\mathrm{b}}\right), 8.82\left(\mathrm{~d}, 2 \mathrm{H},{ }^{3} J_{\mathrm{H}-\mathrm{H}}=4.9 \mathrm{~Hz}\right.$, pyrrole $\left.{ }^{\mathrm{a}}\right), 8.80(\mathrm{~d}$, $2 \mathrm{H},{ }^{3} J_{\mathrm{H}-\mathrm{H}}=4.9 \mathrm{~Hz}$, pyrrole $\left.{ }^{\mathrm{b}}\right), 8.81\left(\mathrm{~s}, 4 \mathrm{H}\right.$, pyrrole $\left.{ }^{\mathrm{a}}\right), 8.77\left(\mathrm{~s}, 4 \mathrm{H}\right.$, pyrrole $\left.{ }^{\mathrm{b}}\right), 8.13\left(\mathrm{dd}, 2 \mathrm{H},{ }^{4} J_{\mathrm{H}-\mathrm{H}}=2.2 \mathrm{~Hz}\right.$, ${ }^{3} J_{\mathrm{H}-\mathrm{H}}=8.3 \mathrm{~Hz}, o$-phenyl $\left.{ }^{\mathrm{a}, \mathrm{b}}\right), 8.03\left(\mathrm{dd}, 1 \mathrm{H},{ }^{4} J_{\mathrm{H}-\mathrm{H}}=2.2 \mathrm{~Hz},{ }^{3} J_{\mathrm{H}-\mathrm{H}}=8.3 \mathrm{~Hz}, o^{\prime}-\right.$ phenyl $\left.\mathrm{b}^{\mathrm{b}}\right), 7.90\left(\mathrm{dd}, 1 \mathrm{H},{ }^{4} J_{\mathrm{H}-}\right.$ ${ }_{\mathrm{H}}=2.2 \mathrm{~Hz},{ }^{3} J_{\mathrm{H}-\mathrm{H}}=8.3 \mathrm{~Hz}, o^{\prime}$-phenyl $\left.\mathrm{l}^{\mathrm{a}}\right), 7.31\left(\mathrm{dd}, 2 \mathrm{H},{ }^{4} J_{\mathrm{H}-\mathrm{H}}=2.2 \mathrm{~Hz},{ }^{3} J_{\mathrm{H}-\mathrm{H}}=8.3 \mathrm{~Hz}, m-\right.$ phenyl $\left.{ }^{\mathrm{a}, \mathrm{b}}\right), 7.17(\mathrm{dd}$, $2 \mathrm{H},{ }^{4} J_{\mathrm{H}-\mathrm{H}}=2.2 \mathrm{~Hz},{ }^{3} J_{\mathrm{H}-\mathrm{H}}=8.3 \mathrm{~Hz}, m^{\prime}$-phenyl ${ }^{\mathrm{a}, \mathrm{b}}$ ), 7.76 (s. $1 \mathrm{H}, o^{\prime \prime}$-phenyl), 7.23 (s. $3 \mathrm{H}, m^{\prime \prime \prime}$-phenyl ${ }^{\mathrm{a}}$ ), 7.21 (s. 3H, $m^{\prime \prime \prime}-$ phenyl ${ }^{\mathrm{b}}$ ), 7.10 (s. 3H, $m^{\prime \prime \prime \prime}$-phenyl ${ }^{\mathrm{a}}$ ), 7.08 (s. 3H, $m^{\prime \prime \prime \prime}-$-phenyl $), 7.46$ (m, 2H, $o^{\prime \prime \prime}-$ phenyl $\left.{ }^{\mathrm{a}, \mathrm{b}}\right), 7.35\left(\mathrm{~m}, 1 \mathrm{H}, o^{\prime \prime \prime \prime}\right.$-phenyl $\left.{ }^{\mathrm{a}, \mathrm{b}}\right), 5.01\left(\mathrm{~s}, 2 \mathrm{H},-\mathrm{OCH}_{2}-^{\mathrm{b}}\right), 5.00\left(\mathrm{~s}, 2 \mathrm{H},-\mathrm{OCH}_{2}{ }^{\mathrm{a}}\right), 2.45(\mathrm{~s}, 9 \mathrm{H}$, $\left.p-\mathrm{CH}_{3}{ }^{\mathrm{a}}\right), 2.44\left(\mathrm{~s}, 9 \mathrm{H}, p-\mathrm{CH}_{3}{ }^{\mathrm{b}}\right), 2.29\left(\mathrm{~s}, 6 \mathrm{H}, o-\mathrm{CH}_{3}{ }^{\mathrm{a}}\right), 2.27\left(\mathrm{~s}, 6 \mathrm{H}, o-\mathrm{CH}_{3}{ }^{\mathrm{b}}\right), 2.05\left(\mathrm{~s}, 3 \mathrm{H} o^{\prime}-\mathrm{CH}_{3}{ }^{\mathrm{a}}\right)$, $2.17\left(\mathrm{~s}, 3 \mathrm{H} o^{\prime}-\mathrm{CH}_{3}{ }^{\mathrm{b}}\right), 1.87\left(\mathrm{~s}, 3 \mathrm{H}, o^{\prime \prime}-\mathrm{CH}_{3}{ }^{\mathrm{a}}\right), 1.78\left(\mathrm{~s}, 3 \mathrm{H}, o^{\prime \prime}-\mathrm{CH}_{3}{ }^{\mathrm{b}}\right), 1.76\left(\mathrm{~s}, 12 \mathrm{H}, o^{\prime \prime \prime}-\mathrm{CH}_{3}\right),-1.61$ 
$\left(\mathrm{dd}, 2 \mathrm{H},{ }^{3} J_{\mathrm{H}-\mathrm{H}}=8.2 \mathrm{~Hz},{ }^{2} J_{103_{\mathrm{Rh}-\mathrm{H}}}=3.4 \mathrm{~Hz}, \mathrm{Rh}-\mathrm{CH}_{2} \mathrm{OH}\right),-4.16\left(\mathrm{t} .1 \mathrm{H},{ }^{3} J_{\mathrm{H}-\mathrm{H}}=8.2 \mathrm{~Hz}, \mathrm{Rh}_{-} \mathrm{CH}_{2} \mathrm{OH}\right),-$ $40.06\left(\mathrm{~d}, 1 \mathrm{H},{ }^{1} J_{103_{\mathrm{Rh}-\mathrm{H}}}=42.8 \mathrm{~Hz}, \mathrm{Rh}-\mathrm{H}\right)$.

${ }^{\mathrm{a}} \mathbf{R h}(\mathbf{m}-\mathbf{x y l y l}) \mathbf{R h}^{\mathrm{b}}-\mathbf{C H}_{2} \mathbf{O H}(\mathbf{1 1}):{ }^{1} \mathrm{H}$ NMR $\left(\mathrm{C}_{6} \mathrm{D}_{6}, 296 \mathrm{~K}\right), \delta(\mathrm{ppm}): 5.90\left(\mathrm{~s}, 2 \mathrm{H},-\mathrm{OCH}_{2}{ }^{-}{ }^{\mathrm{a}}\right)$, $5.31\left(\mathrm{~s}, 2 \mathrm{H},-\mathrm{OCH}_{2}{ }^{-}{ }^{\mathrm{b}}\right),-1.60\left(\mathrm{dd}, 2 \mathrm{H},{ }^{3} J_{\mathrm{H}-\mathrm{H}}=8.2 \mathrm{~Hz},{ }^{2} J_{103_{\mathrm{Rh}-\mathrm{H}}}=3.4 \mathrm{~Hz}, \mathrm{Rh}-\mathrm{CH}_{2} \mathrm{OH}\right),-4.15$ (t. $1 \mathrm{H},{ }^{3} J_{\mathrm{H}-}$ $\left.{ }_{\mathrm{H}}=8.2 \mathrm{~Hz}, \mathrm{Rh}_{-} \mathrm{CH}_{2} \mathrm{OH}\right)$.

$\mathbf{H O C H}_{2}-\mathbf{R h}(\mathbf{m}-\mathbf{x y l y l}) \mathbf{R h}-\mathbf{C H}_{2} \mathbf{O H}(\mathbf{1 2}):{ }^{1} \mathrm{H}$ NMR $\left(\mathrm{C}_{6} \mathrm{D}_{6}, 296 \mathrm{~K}\right), \delta(\mathrm{ppm}): 8.97\left(\mathrm{~d}, 4 \mathrm{H},{ }^{3} J_{\mathrm{H}-}\right.$ ${ }_{\mathrm{H}}=4.9 \mathrm{~Hz}$, pyrrole $), 8.80\left(\mathrm{~d}, 4 \mathrm{H},{ }^{3} J_{\mathrm{H}-\mathrm{H}}=4.9 \mathrm{~Hz}\right.$, pyrrole $), 8.77(\mathrm{~s}, 8 \mathrm{H}$, pyrrole $), 8.14\left(\mathrm{dd}, 2 \mathrm{H},{ }^{4} J_{\mathrm{H}-}\right.$ ${ }_{\mathrm{H}}=2.2 \mathrm{~Hz},{ }^{3} J_{\mathrm{H}-\mathrm{H}}=8.3 \mathrm{~Hz}, o$-phenyl), $8.03\left(\mathrm{dd}, 2 \mathrm{H},{ }^{4} J_{\mathrm{H}-\mathrm{H}}=2.2 \mathrm{~Hz},{ }^{3} J_{\mathrm{H}-\mathrm{H}}=8.3 \mathrm{~Hz}, o^{\prime}\right.$-phenyl), 7.32 (dd, $2 \mathrm{H}$, ${ }^{4} J_{\mathrm{H}-\mathrm{H}}=2.2 \mathrm{~Hz},{ }^{3} J_{\mathrm{H}-\mathrm{H}}=8.3 \mathrm{~Hz}, m$-phenyl), 7.17 (dd, $2 \mathrm{H},{ }^{4} J_{\mathrm{H}-\mathrm{H}}=2.2 \mathrm{~Hz},{ }^{3} J_{\mathrm{H}-\mathrm{H}}=8.3 \mathrm{~Hz}, m^{\prime}$-phenyl), 7.77 (s. 1H, $o^{\prime \prime}$-phenyl), 7.22 (s. 6H, m"'-phenyl), 7.08 (s. 6H, $m^{\prime \prime \prime \prime}$-phenyl), 7.46 (m, 2H, $o^{\prime \prime \prime}$-phenyl), 7.35 (m, 1H, $o^{\prime \prime \prime \prime}$-phenyl), $5.01\left(\mathrm{~s}, 4 \mathrm{H},-\mathrm{OCH}_{2}-\right), 2.44\left(\mathrm{~s}, 18 \mathrm{H}, p-\mathrm{CH}_{3}\right), 2.27\left(\mathrm{~s}, 12 \mathrm{H}, o-\mathrm{CH}_{3}\right), 2.17(\mathrm{~s}, 6 \mathrm{H}$ $\left.o^{\prime}-\mathrm{CH}_{3}\right), 1.78\left(\mathrm{~s}, 6 \mathrm{H}, o^{\prime \prime}-\mathrm{CH}_{3}\right), 1.76\left(\mathrm{~s}, 12 \mathrm{H}, o^{\prime \prime \prime}-\mathrm{CH}_{3}\right),-1.60\left(\mathrm{dd}, 4 \mathrm{H},{ }^{3} J_{\mathrm{H}-\mathrm{H}}=8.2 \mathrm{~Hz},{ }^{2} J_{103 \mathrm{Rh}-\mathrm{H}}=3.4 \mathrm{~Hz}\right.$, Rh- $\mathrm{CH}_{2} \mathrm{OH}$ ), -4.13 (t. $2 \mathrm{H},{ }^{3} \mathrm{~J}_{\mathrm{H}-\mathrm{H}}=8.2 \mathrm{~Hz}, \mathrm{Rh}_{-} \mathrm{CH}_{2} \mathrm{OH}$ ).

$\mathbf{C}_{6} \mathbf{H}_{5} \mathbf{C H}_{2}-\mathbf{R h}(\mathbf{m}-\mathbf{x y l y l}) \mathbf{R h}-\mathbf{C H}_{2} \mathbf{C}_{6} \mathbf{H}_{5}(\mathbf{1 3}):{ }^{1} \mathrm{H}$ NMR $\left(\mathrm{C}_{6} \mathrm{D}_{6}\right), \delta(\mathrm{ppm}) 8.83\left(\mathrm{~d}, 4 \mathrm{H},{ }^{3} J_{\mathrm{H}-\mathrm{H}}=\right.$ $5.0 \mathrm{~Hz}$, pyrrole), $8.81\left(\mathrm{~d}, 4 \mathrm{H},{ }^{3} J_{\mathrm{H}-\mathrm{H}}=5.0 \mathrm{~Hz}\right.$, pyrrole $), 8.78\left(\mathrm{~d}, 4 \mathrm{H},{ }^{3} J_{\mathrm{H}-\mathrm{H}}=5.0 \mathrm{~Hz}\right.$, pyrrole $), 8.73(\mathrm{~d}$, $4 \mathrm{H},{ }^{3} J_{\mathrm{H}-\mathrm{H}}=5.0 \mathrm{~Hz}$, pyrrole), $8.03\left(\mathrm{dd}, 2 \mathrm{H},{ }^{3} J_{\mathrm{H}-\mathrm{H}}=8.5 \mathrm{~Hz},{ }^{4} J_{\mathrm{H}-\mathrm{H}}=1.9 \mathrm{~Hz}, \mathrm{o}-\right.$ phenyl), $7.95\left(\mathrm{dd}, 2 \mathrm{H},{ }^{3} J_{\mathrm{H}-}\right.$ ${ }_{\mathrm{H}}=8.5 \mathrm{~Hz},{ }^{4} J_{\mathrm{H}-\mathrm{H}}=1.9 \mathrm{~Hz}, \mathrm{o}^{\prime}-$ phenyl $), 7.78\left(\mathrm{~s}, 1 \mathrm{H}\right.$, o-spacer-phenyl), $7.48\left(\mathrm{~d}, 2 \mathrm{H},{ }^{3} J_{\mathrm{H}-\mathrm{H}}=7.0 \mathrm{~Hz}, \mathrm{o}^{\prime}-\right.$ spacer-phenyl), 7.37(t, $1 \mathrm{H} .{ }^{3} J_{\mathrm{H}-\mathrm{H}}=7.0 \mathrm{~Hz}, \mathrm{~m}$-spacer-phenyl), 7.34(dd, $2 \mathrm{H},{ }^{3} J_{\mathrm{H}-\mathrm{H}}=8.3 \mathrm{~Hz},{ }^{4} J_{\mathrm{H}-\mathrm{H}}=2.6$ $\mathrm{Hz}, \mathrm{m}$-phenyl), 7.17(dd, $2 \mathrm{H},{ }^{3} J_{\mathrm{H}-\mathrm{H}}=8.3 \mathrm{~Hz},{ }^{4} J_{\mathrm{H}-\mathrm{H}}=2.6 \mathrm{~Hz}, \mathrm{~m}$-phenyl), $7.20(\mathrm{~s}, 6 \mathrm{H}, \mathrm{m}$ "-phenyl), 7.20(s, 2H, m"'-phenyl), 7.11(s, 2H, m"'--phenyl), 6.93(s, 6H, m"'"-phenyl), 5.04(s, 4H, - $\mathrm{OCH}_{2}-$ ), $2.47\left(\mathrm{~s}, 18 \mathrm{H}, \mathrm{p}-\mathrm{CH}_{3}\right), 2.44\left(\mathrm{~s}, 12 \mathrm{H}, \mathrm{o}-\mathrm{CH}_{3}\right), 2.17\left(\mathrm{~s}, 6 \mathrm{H}, \mathrm{o}^{\prime}-\mathrm{CH}_{3}\right), 1.82\left(\mathrm{~s}, 6 \mathrm{H}, \mathrm{o}^{\prime \prime}-\mathrm{CH}_{3}\right), 2.10(\mathrm{~s}, 12 \mathrm{H}$, o'"-- $\left.\mathrm{CH}_{3}\right), 6.40\left(\mathrm{t}, 2 \mathrm{H}, \mathrm{p}\right.$-benzyl, $\left.{ }^{3} J_{\mathrm{H}-\mathrm{H}}=7.8 \mathrm{~Hz}\right), 5.89\left(\mathrm{t}, 4 \mathrm{H}, \mathrm{m}-\right.$ benzyl, $\left.{ }^{3} J_{\mathrm{H}-\mathrm{H}}=7.8,7.4 \mathrm{~Hz}\right), 3.48(\mathrm{~d}$, $4 \mathrm{H}, \mathrm{o}-$ benzyl, $\left.{ }^{3} \mathrm{~J}_{\mathrm{H}-\mathrm{H}}=7.4 \mathrm{~Hz}\right),-3.21\left(\mathrm{~d}, 4 \mathrm{H}, \mathrm{Rh}-\mathrm{CH}_{2} \mathrm{C}_{6} \mathrm{H}_{5},{ }^{2} J_{103_{\mathrm{Rh}-\mathrm{H}}}=3.7 \mathrm{~Hz}\right)$. 
${ }^{\mathrm{a}} \mathbf{R h}(\mathbf{m}-\mathbf{x y l y l}) \mathbf{R h}^{\mathrm{b}}-\mathrm{CH}_{2} \mathbf{C}_{6} \mathbf{H}_{5}(\mathbf{1 4}):{ }^{1} \mathrm{H}$ NMR $\left(\mathrm{C}_{6} \mathrm{D}_{6}, 296 \mathrm{~K}\right), \delta(\mathrm{ppm}): 5.90\left(\mathrm{~s}, 2 \mathrm{H},-\mathrm{OCH}_{2}-\right.$ $\left.{ }^{\mathrm{a}}\right), 5.36\left(\mathrm{~s}, 2 \mathrm{H},-\mathrm{OCH}_{2}{ }^{-}{ }^{\mathrm{b}}\right),-3.17\left(\mathrm{~d}, 2 \mathrm{H}, \mathrm{Rh}-\mathrm{CH}_{2} \mathrm{C}_{6} \mathrm{H}_{5},{ }^{2} \mathrm{~J}_{103_{\mathrm{Rh}} \mathrm{H}}=3.7 \mathrm{~Hz}\right)$.

$\mathbf{H}-\mathbf{R h}{ }^{\mathrm{a}}(\mathbf{m}-\mathbf{x y l y l}) \mathbf{R h}^{\mathrm{b}}-\mathbf{C H}_{2} \mathbf{C}_{6} \mathbf{H}_{5}(\mathbf{1 5}):{ }^{1} \mathrm{H}$ NMR $\left(\mathrm{C}_{6} \mathrm{D}_{6}, 296 \mathrm{~K}\right), \delta(\mathrm{ppm}): 8.95\left(\mathrm{~d}, 2 \mathrm{H},{ }^{3} J_{\mathrm{H}-}\right.$ ${ }_{\mathrm{H}}=4.9 \mathrm{~Hz}$, pyrrole $), 8.83\left(\mathrm{~d}, 2 \mathrm{H},{ }^{3} J_{\mathrm{H}-\mathrm{H}}=5.0 \mathrm{~Hz}\right.$, pyrrole $), 8.82\left(\mathrm{~d}, 2 \mathrm{H},{ }^{3} J_{\mathrm{H}-\mathrm{H}}=4.9 \mathrm{~Hz}\right.$, pyrrole $), 8.81(\mathrm{~d}$, $2 \mathrm{H},{ }^{3} J_{\mathrm{H}-\mathrm{H}}=5.0 \mathrm{~Hz}$, pyrrole $\left.^{\mathrm{b}}\right), 8.80\left(\mathrm{~s}, 4 \mathrm{H}\right.$, pyrrole $\left.{ }^{\mathrm{a}}\right), 8.78\left(\mathrm{~d}, 2 \mathrm{H},{ }^{3} J_{\mathrm{H}-\mathrm{H}}=5.0 \mathrm{~Hz}\right.$, pyrrole $\left.^{\mathrm{b}}\right), 8.73(\mathrm{~d}, 2 \mathrm{H}$, ${ }^{3} J_{\mathrm{H}-\mathrm{H}}=5.0 \mathrm{~Hz}$, pyrrole $\left.{ }^{\mathrm{b}}\right), 8.13\left(\mathrm{dd}, 1 \mathrm{H},{ }^{4} J_{\mathrm{H}-\mathrm{H}}=2 \mathrm{~Hz},{ }^{3} J_{\mathrm{H}-\mathrm{H}}=8.3 \mathrm{~Hz}, o\right.$-phenyl $\left.{ }^{\mathrm{a}}\right), 8.03\left(\mathrm{dd}, 1 \mathrm{H},{ }^{4} J_{\mathrm{H}-\mathrm{H}}=2 \mathrm{~Hz}\right.$, ${ }^{3} J_{\mathrm{H}-\mathrm{H}}=8.3 \mathrm{~Hz}, o$-phenyl $\left.{ }^{\mathrm{b}}\right), 7.90\left(\mathrm{dd}, 1 \mathrm{H},{ }^{4} J_{\mathrm{H}-\mathrm{H}}=2.2 \mathrm{~Hz},{ }^{3} J_{\mathrm{H}-\mathrm{H}}=8.3 \mathrm{~Hz}, o^{\prime}\right.$-phenyl $\left.{ }^{\mathrm{a}}\right), 7.95\left(\mathrm{dd}, 1 \mathrm{H},{ }^{4} J_{\mathrm{H}-}\right.$ ${ }_{\mathrm{H}}=2.2 \mathrm{~Hz},{ }^{3} J_{\mathrm{H}-\mathrm{H}}=8.3 \mathrm{~Hz}, o^{\prime}-$ phenyl $\left.{ }^{\mathrm{b}}\right), 7.31\left(\mathrm{dd}, 1 \mathrm{H},{ }^{4} J_{\mathrm{H}-\mathrm{H}}=2.2 \mathrm{~Hz},{ }^{3} J_{\mathrm{H}-\mathrm{H}}=8.3 \mathrm{~Hz}, m-\right.$ phenyl $\left.{ }^{\mathrm{a}}\right), 7.34(\mathrm{dd}$, $1 \mathrm{H},{ }^{4} J_{\mathrm{H}-\mathrm{H}}=2.2 \mathrm{~Hz},{ }^{3} J_{\mathrm{H}-\mathrm{H}}=8.3 \mathrm{~Hz}, m-$ phenyl $\left.{ }^{\mathrm{b}}\right), 7.17\left(\mathrm{dd}, 2 \mathrm{H},{ }^{4} J_{\mathrm{H}-\mathrm{H}}=2.2 \mathrm{~Hz},{ }^{3} J_{\mathrm{H}-\mathrm{H}}=8.3 \mathrm{~Hz}, m^{\prime}\right.$-phenyl $\left.{ }^{\mathrm{a}, \mathrm{b}}\right)$, 7.76 (s. 1H, o-spacer-phenyl), 7.45 (m, 2H, $o^{\prime}$-spacer-phenyl), 7.36 (m, 1H, $m$-spacer-phenyl), 7.22 (s. 3H, $m^{\prime \prime}$-phenyl $\mathrm{l}^{\mathrm{a}}$ ), 7.03 (s. 3H, $m^{\prime \prime}$-phenyl $\mathrm{l}^{\mathrm{b}}$ ), 7.10 (s. 3H, $\mathrm{m}^{\prime \prime \prime}$-phenyl $\mathrm{l}^{\mathrm{a}}$ ), 6.93 (s. $3 \mathrm{H}, \mathrm{m}^{\prime \prime \prime}$ phenyl $\left.{ }^{\mathrm{b}}\right), 4.99\left(\mathrm{~s}, 2 \mathrm{H},-\mathrm{OCH}_{2^{-}}{ }^{\mathrm{a}}\right), 5.04\left(\mathrm{~s}, 2 \mathrm{H},-\mathrm{OCH}_{2}{ }^{-}{ }^{\mathrm{b}}\right), 2.43\left(\mathrm{~s}, 9 \mathrm{H}, p-\mathrm{CH}_{3}{ }^{\mathrm{a}}\right), 2.47\left(\mathrm{~s}, 9 \mathrm{H}, p-\mathrm{CH}_{3}{ }^{\mathrm{b}}\right)$, $2.29\left(\mathrm{~s}, 6 \mathrm{H}, o-\mathrm{CH}_{3}{ }^{\mathrm{a}}\right), 2.44\left(\mathrm{~s}, 6 \mathrm{H}, o-\mathrm{CH}_{3}{ }^{\mathrm{b}}\right), 2.05\left(\mathrm{~s}, 3 \mathrm{H} o^{\prime}-\mathrm{CH}_{3}{ }^{\mathrm{a}}\right), 2.17\left(\mathrm{~s}, 3 \mathrm{H} o^{\prime}-\mathrm{CH}_{3}{ }^{\mathrm{b}}\right), 1.87(\mathrm{~s}$, $\left.3 \mathrm{H}, o^{\prime \prime}-\mathrm{CH}_{3}{ }^{\mathrm{a}}\right), 1.82\left(\mathrm{~s}, 3 \mathrm{H}, o^{\prime \prime}-\mathrm{CH}_{3}{ }^{\mathrm{b}}\right), 1.76\left(\mathrm{~s}, 6 \mathrm{H}, o^{\prime \prime \prime}-\mathrm{CH}_{3}{ }^{\mathrm{a}}\right), 2.10\left(\mathrm{~s}, 6 \mathrm{H}, o^{\prime \prime \prime}-\mathrm{CH}_{3}{ }^{\mathrm{b}}\right), 6.40(\mathrm{t}, 1 \mathrm{H}, \mathrm{p}-$ benzyl, $\left.{ }^{3} J_{\mathrm{H}-\mathrm{H}}=7.8 \mathrm{~Hz}\right), 5.89\left(\mathrm{t}, 2 \mathrm{H}, \mathrm{m}-\right.$ benzyl, $\left.{ }^{3} J_{\mathrm{H}-\mathrm{H}}=7.8,7.4 \mathrm{~Hz}\right), 3.48\left(\mathrm{~d}, 2 \mathrm{H}\right.$, o-benzyl, ${ }^{3} J_{\mathrm{H}-\mathrm{H}}=7.4$ $\mathrm{Hz}),-3.20\left(\mathrm{~d}, 2 \mathrm{H}, \mathrm{Rh}-\mathrm{CH}_{2} \mathrm{C}_{6} \mathrm{H}_{5},{ }^{2} J_{103_{\mathrm{Rh}-\mathrm{H}}}=3.7 \mathrm{~Hz}\right),-40.06\left(\mathrm{~d}, 1 \mathrm{H},{ }^{1} J_{103_{\mathrm{Rh}-\mathrm{H}}}=42.5 \mathrm{~Hz}, \mathrm{Rh}-H\right)$.

Reaction of $\cdot \mathbf{R h}(\mathbf{m}-\mathbf{x y l y}) \mathbf{R h} \cdot$ with Methane: Benzene solutions of $\cdot \operatorname{Rh}(m-x y l y l) R h \cdot(\mathbf{1})$ react with methane $\left(\mathrm{P}_{\mathrm{CH}_{4}} \sim 0.2-0.8 \mathrm{~atm},\left[\mathrm{CH}_{4}\right] \sim 4.5 \times 10^{-3}-1.9 \times 10^{-2} \mathrm{M}, \mathrm{T}=296 \mathrm{~K}\right)$ to form species with Rh-H and $\mathrm{Rh}_{-} \mathrm{CH}_{3}$ units as evidenced by observation of characteristic high field ${ }^{1} \mathrm{H}$ NMR resonances $\quad\left(\delta_{\mathrm{Rh}-\mathrm{H}}=-40.06 \mathrm{ppm},{ }^{1} J_{103_{\mathrm{Rh}-\mathrm{H}}}=42.5 \mathrm{~Hz} ; \delta_{\mathrm{Rh}_{-} \mathrm{CH}_{3}}=-5.29 \mathrm{ppm},{ }^{2} J_{103_{\mathrm{Rh}-\mathrm{H}}}=2.9 \mathrm{~Hz}\right)$. During the early phase of the reaction when observable quantities of the dimetalloradical $\mathbf{1}$ are still present, two Rh- $\mathrm{CH}_{3}\left(\delta_{\mathrm{Rh}_{-} \mathrm{CH}_{3}}=-5.27,-5.29 \mathrm{ppm}\right)$ and two Rh- $H\left(\delta_{\mathrm{Rh}-\mathrm{H}}=-40.03,-40.06 \mathrm{ppm}\right)$ resonances are observed. A pair of spacer $\mathrm{CH}_{2}$ peaks $\left(\delta_{\mathrm{CH}_{2}}=5.90\right.$ and $\left.5.31 \mathrm{ppm}\right)$ along with high field resonances $\left(\delta_{\mathrm{Rh}_{-\mathrm{CH}}}=-5.27 \mathrm{ppm}, \delta_{\mathrm{Rh}-\mathrm{H}}=-40.03 \mathrm{ppm}\right)$ in a near 3:1 intensity ratio are assigned to $\cdot \mathrm{Rh}(\mathrm{m}-\mathrm{xylyl}) \mathrm{Rh}-\mathrm{H}(3)$ and $\cdot \mathrm{Rh}(\mathrm{m}-\mathrm{xylyl}) \mathrm{Rh}-\mathrm{CH}_{3}$ (5) in effectively equal quantities. The 
remaining peaks $\left(\delta_{\mathrm{CH}_{2}}=5.00 \mathrm{ppm}, \delta_{\mathrm{Rh}_{-} \mathrm{CH}_{3}}=-5.29 \mathrm{ppm}, \delta_{\mathrm{Rh}-\mathrm{H}}=-40.06 \mathrm{ppm}\right)$ are characteristic of diamagnetic species that contain $\mathrm{Rh}-\mathrm{H}$ and $\mathrm{Rh}-\mathrm{CH}_{3}$ units. As the paramagnetic species $(\mathbf{1}, \mathbf{3}$ and $\mathbf{5})$ react away, diamagnetic species with a near equal numbers of $\mathrm{Rh}-\mathrm{H}$ and $\mathrm{Rh}-\mathrm{CH}_{3}$ units are formed. Using only ${ }^{1} \mathrm{H}$ NMR it is difficult to distinguish between the presence of a single diamagnetic species $\mathrm{H}-\mathrm{Rh}(\mathrm{m}-\mathrm{xylyl}) \mathrm{Rh}-\mathrm{CH}_{3}(\mathbf{6})$ and a mixture of $\mathbf{2}, \mathbf{4}$ and $\mathbf{6}$, because the tether $\mathrm{CH}_{2}$ is a singlet at $\sim 5.00 \mathrm{ppm}$ in each species. At the time when most of the paramagnetic species $(\mathbf{1}, \mathbf{3}$ and $\mathbf{5})$ have reacted to produce diamagnetic species the FAB mass spectra for the solution has a dominant parent ion peak for a mono-methyl species (1833.3D; $\left.\mathrm{H}-\mathrm{Rh}(\mathrm{m}-\mathrm{xylyl}) \mathrm{Rh}-\mathrm{CH}_{3}(1833.9 \mathrm{D})\right)$ and the dimethyl derivative (4) $\left(\mathrm{H}_{3} \mathrm{C}-\mathrm{Rh}\right.$ (m-xylyl) $\left.\mathrm{Rh}-\mathrm{CH}_{3}, 1847.9 \mathrm{D}\right)$ is not detected. The mono-methyl derivative is shown to occur as the exclusive diamagnetic product with a $\mathrm{Rh}-\mathrm{CH}_{3}$ unit that is formed during the time needed for most of the paramagmetic species $(\mathbf{1}, \mathbf{3}$ and $\mathbf{5})$ to react away (eq. S1). Intermolecular reactions involving $\mathrm{Rh}^{\mathrm{II}}$. centers in different (S1)

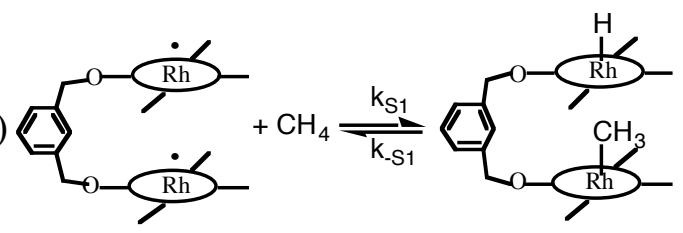

molecular units and methyl transfer between $\mathrm{Rh}_{-} \mathrm{CH}_{3}$ and $\mathrm{Rh}^{\mathrm{I}}$. sites that are needed to produce the dimethyl complex $\mathbf{4}$ must be very slow compared to reactions that use two $\mathrm{Rh}^{\mathrm{II}}$. centers in the same molecule that exclusively produce $\mathrm{H}-\mathrm{Rh}(\mathrm{m}-\mathrm{xylyl}) \mathrm{Rh}-\mathrm{CH}_{3}$. The paramagnetic monohydride and mono-methyl species that occur in conjunction with $\mathrm{H}-\mathrm{Rh}(\mathrm{m}$-xylyl $) \mathrm{Rh}_{-} \mathrm{CH}_{3}(6)$ result from reversible hydrogen atom transfer from 6 to $\cdot \mathrm{Rh}(\mathrm{m}-\mathrm{xylyl}) \mathrm{Rh} \cdot(\mathbf{1})$ and not from either the intermolecular use of $\mathrm{Rh}^{\mathrm{II}}$. sites in the reaction of two molecules of $\mathbf{1}$ with methane or interchange of $\mathrm{CH}_{3}$ groups between $\mathrm{Rh}-\mathrm{CH}_{3}$ and $\mathrm{Rh}^{\mathrm{II}}$. centers.

Integration of the ${ }^{1} \mathrm{H}$ NMR over several days showed that the number of Rh-H sites regularly decreases, and the number of $\mathrm{Rh}-\mathrm{CH}_{3}$ sites increases among the reaction products. The reaction system consisting of $\mathbf{1}$ and methane is ultimately transformed to $\mathrm{H}_{3} \mathrm{C}-\mathrm{Rh}\left(\mathrm{m}\right.$-xylyl) $\mathrm{Rh}-\mathrm{CH}_{3}$ and $\mathrm{H}_{2}$. FAB mass spectra for the methane reaction solutions after a period of several days or longer (296K) manifest a substantial parent ion peak for the dimethyl complex (4) (1847.4D). Hydrogen 
atom transfer between $\mathrm{Rh}^{\mathrm{I}}$. centers, reductive elimination of $\mathrm{H}_{2}$ and slow intermolecular oxidative addition and reductive elimination of methane permit the reaction system to move slowly toward the formation of $\mathrm{H}_{3} \mathrm{C}-\mathrm{Rh}(\mathrm{m}-\mathrm{xylyl}) \mathrm{Rh}-\mathrm{CH}_{3}$ (4).

Kinetics for the reaction of $\cdot \mathrm{Rh}(\mathrm{m}$-xylyl $) \mathrm{Rh} \cdot$ with $\mathrm{CH}_{4}$ were obtained by monitoring the change of the tether methylene ${ }^{1} \mathrm{H}$ NMR peak of $\mathbf{1}(6.21 \mathrm{ppm})$ and the appearance of the peaks associated with 3,5 and $\mathbf{6}$ over a range of conditions $\left(\mathrm{P}_{\mathrm{CH}_{4}}=100-600\right.$ torr; $\mathrm{T}=274-305 \mathrm{~K} ;[\mathbf{1}]=$ $\left.3.0 \times 10^{-3}-2.5 \times 10^{-2} \mathrm{M}\right)$. The methane concentration in solution and the reservoir of methane gas are large compared to the concentration of $\mathbf{1}$, which ensures that the observed kinetics for each single sample is pseudo zero order in methane. Variation of the methane concentration was used to determine that the initial reaction of $\mathbf{1}$ with methane is first order in substrate.

The kinetics for the disappearance of $\mathbf{1}$ by reaction with $\mathrm{CH}_{4}$ was fitted to a bimolecular process $\left(\right.$ rate $_{\mathrm{S} 1}=\mathrm{k}_{\mathrm{S} 1}(\mathrm{app})[\mathbf{1}]\left[\right.$ Substrate]) with an apparent second-order rate constant $\mathrm{k}_{\mathrm{S} 1}(\operatorname{app})$ of $0.105 \mathrm{M}^{-1} \mathrm{~s}^{-1}$ (Figure S1). The derived rate constant is an order of magnitude larger than the value previously reported $\left(\mathrm{k}=8.6 \times 10^{-3} \mathrm{M}^{-1} \mathrm{~s}^{-1}\right)$ for reaction of methane with a six-carbon chain tethered dirhodium(II) species $^{3}$ and the reaction rate for $\mathbf{1}$ is many orders of magnitude larger than the reaction rate for $\mathrm{CH}_{4}$ with (TMP)Rh. ${ }^{2}$ (Figure S1). Kinetic studies for reactions of $\mathbf{1}$ with deuterated methane were used in evaluating the kinetic isotope effect for reaction S1 at $296 \mathrm{~K}$ $\left(\mathrm{k}_{\mathrm{S} 1}\left(\mathrm{CD}_{4}\right)(\mathrm{app})=9.7 \times 10^{-3} \mathrm{M}^{-1} \mathrm{~s}^{-1}, \mathrm{k}_{\mathrm{S} 1}\left(\mathrm{CH}_{4}\right) / \mathrm{k}_{\mathrm{S} 1}\left(\mathrm{CD}_{4}\right)=10.8 \pm 1.0\right)^{4}$.

Apparent activation parameters $\left(\Delta \mathrm{H}_{\mathrm{S} 1}{ }^{\ddagger}(\mathrm{app})=9.8 \pm 0.5 \mathrm{kcal} \mathrm{mol}^{-1} ; \Delta \mathrm{S}_{\mathrm{S} 1}{ }^{\ddagger}(\mathrm{app})=-30 \pm 3\right.$ cal $\mathrm{K}^{-1} \mathrm{~mol}^{-1}$ ) were derived from the temperature dependence of the apparent second order rate constants $\left(\mathrm{k}_{\mathrm{S} 1}(\mathrm{app})\right)$ for the $\mathrm{C}-\mathrm{H}$ bond reactions of $\mathbf{1}$ with $\mathrm{CH}_{4} \cdot{ }^{4}$ 


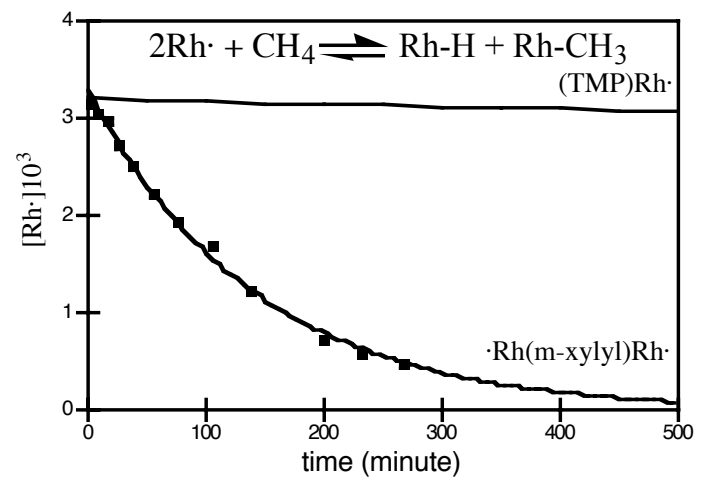

Figure S1. Change in the $[\mathrm{Rh} \cdot]$ center with the time for the reaction of methane $\left(\mathrm{P}_{\mathrm{CH}_{4}}=202\right.$ torr, $\left.\left[\mathrm{CH}_{4}\right]=6.3 \times 10^{-3} \mathrm{M}\right)$ with $(\mathrm{TMP}) \mathrm{Rh} \cdot\left(\mathrm{k}=0.132 \mathrm{M}^{-2} \mathrm{~s}^{-1}\right)$ and $\cdot \mathrm{Rh}(\mathrm{m}-\mathrm{xylyl}) \mathrm{Rh} \cdot\left(\mathrm{k}_{4}(\mathrm{app})=0.105 \mathrm{M}^{-}\right.$ $\left.{ }^{1} \mathrm{~s}^{-1}\right)$ in $\mathrm{C}_{6} \mathrm{D}_{6}$ at $296 \mathrm{~K}\left([(\mathrm{TMP}) \mathrm{Rh} \cdot]_{\mathrm{i}}=3.2 \times 10^{-3} \mathrm{M} ;[\cdot \mathrm{Rh}(\mathrm{m}-\mathrm{xylyl}) \mathrm{Rh} \cdot]_{\mathrm{i}}=1.6 \times 10^{-3} \mathrm{M}\right)$

The bimolecular reaction of $\mathbf{1}$ with methane is relatively fast and achieves an equilibrium distribution between $\cdot \mathrm{Rh}\left(\mathrm{m}\right.$-xylyl)Rh$\cdot \mathrm{H}-\mathrm{Rh}(\mathrm{m}-\mathrm{xylyl}) \mathrm{Rh}-\mathrm{CH}_{3}$ and $\mathrm{CH}_{4}$, which then slowly react on together as an equilibrium distribution. The equilibrium constant for reaction S1 was evaluated for a series of samples at low $\mathrm{CH}_{4}$ pressure $\left(\mathrm{P}_{\mathrm{CH}_{4}}=20\right.$ - 30torr) by integration of the ${ }^{1} \mathrm{H}$ NMR for $\mathbf{1}, \mathbf{6}$ and $\left.\mathrm{CH}_{4}\left(\mathrm{~K}_{\mathrm{S} 1}(296 \mathrm{~K})=8.0 \pm 0.2\right) \times 10^{3} ; \Delta \mathrm{G}_{\mathrm{S} 1}{ }^{\circ}(296 \mathrm{~K})=-5.4 \pm 0.1 \mathrm{kcal} \mathrm{mol}^{-1}\right)$.

Kinetic studies for reactions of $\mathbf{1}$ with methane and deuterated methane were used in evaluating the kinetic isotope effect at $296 \mathrm{~K}\left(\mathrm{k}_{\mathrm{S} 1}\left(\mathrm{CH}_{4}\right)(\mathrm{app})=0.105 \mathrm{M}^{-1} \mathrm{~S}^{-1}\right) ; \mathrm{k}_{\mathrm{S} 1}\left(\mathrm{CD}_{4}\right)(\mathrm{app})=9.7$ $\left.\times 10^{-3} \mathrm{M}^{-1} \mathrm{~s}^{-1}\right) ;\left(\mathrm{k}_{\mathrm{S} 1}\left(\mathrm{CH}_{4}\right) / \mathrm{k}_{\mathrm{S} 1}\left(\mathrm{CD}_{4}\right)=10.8 \pm 1.0\right)$. Apparent activation parameters $\left(\Delta \mathrm{H}_{\mathrm{s} 1}{ }^{\ddagger}(\mathrm{app})=9.8\right.$ $\pm 0.5 \mathrm{kcal} \mathrm{mol}^{-1} ; \Delta \mathrm{S}_{\mathrm{S} 1}{ }^{\ddagger}(\mathrm{app})=-30 \pm 3 \mathrm{cal} \mathrm{K}^{-1} \mathrm{~mol}^{-1}$ ) were derived from the temperature dependence of the apparent second order rate constants $\left(\mathrm{k}_{\mathrm{S} 1}(\mathrm{app})\right)$ for the $\mathrm{C}-\mathrm{H}$ bond reactions of $\mathbf{1}$ with $\mathrm{CH}_{4}$ (Figure S2). 
(A)
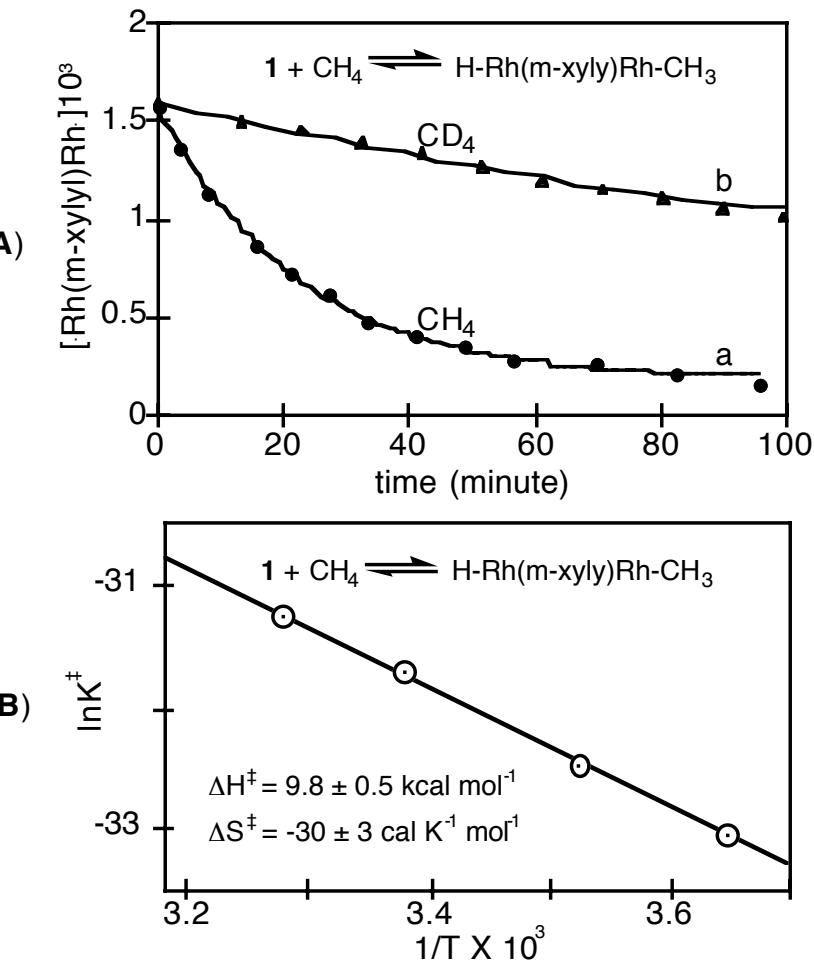

Figure S2. (A) Changes in the $[\cdot \mathrm{Rh}(\mathrm{m}-\mathrm{xylyl}) \mathrm{Rh} \cdot]\left([1]_{\mathrm{i}}=1.6 \times 10^{-3} \mathrm{M}\right)$ in $\mathrm{C}_{6} \mathrm{D}_{6}$ at $296 \mathrm{~K}$ with time in the reaction of: a) $\mathrm{CH}_{4}\left(6.3 \times 10^{-3} \mathrm{M}\right) ; \mathrm{k}_{\mathrm{S} 1}\left(\mathrm{CH}_{4}\right)(\mathrm{app})=0.105 \mathrm{M}^{-1} \mathrm{~s}^{-1}$ and b) $\mathrm{CD}_{4}\left(8.7 \times 10^{-3} \mathrm{M}\right)$; $\mathrm{k}_{\mathrm{s} 1}\left(\mathrm{CD}_{4}\right)(\mathrm{app})=9.7 \times 10^{-3} \mathrm{M}^{-1} \mathrm{~s}^{-1}$; $\quad$ (B) Determination of the activation parameters for reaction of $\cdot \mathrm{Rh}(\mathrm{m}-\mathrm{xylyl}) \mathrm{Rh} \cdot$ with $\mathrm{CH}_{4}$

Reaction of $\cdot \mathbf{R h}(\mathbf{m}-x y l y l) R h \cdot(1)$ with Ethane: Ethane $\left(\mathrm{P}_{\mathrm{C}_{2} \mathrm{H}_{6}}=150-600\right.$ torr, $\left.\left[\mathrm{C}_{2} \mathrm{H}_{6}\right]=2.7 \times 10^{-2}-1.1 \times 10^{-1} \mathrm{M}\right)$ is observed to react with $\mathbf{1}$ at room temperature to form a $\mathrm{C}-\mathrm{H}$ bond activation product assigned as $\mathrm{H}-\mathrm{Rh}(\mathrm{m}-\mathrm{xylyl}) \mathrm{Rh}-\mathrm{CH}_{2} \mathrm{CH}_{3}$ (7) (eq. S2), which is identified by a triplet of doublets at $-3.89 \mathrm{ppm}\left(\mathrm{Rh}-\mathrm{CH}_{2} \mathrm{CH}_{3}\right)$, a quartet of doublets at $-4.33 \mathrm{ppm}\left(\mathrm{Rh}-\mathrm{CH}_{2} \mathrm{CH}_{3}\right)$

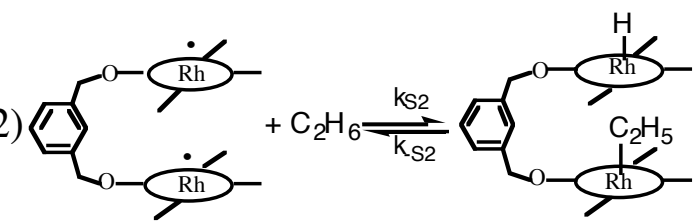

and a doublet at $-40.06 \mathrm{ppm}(\mathrm{Rh}-H)$ in the ${ }^{1} \mathrm{H}$ NMR spectrum. Monorhodium (II) complexes of $\cdot \mathrm{Rh}\left(\mathrm{m}\right.$-xylyl)Rh-H (3), and $\cdot \mathrm{Rh}(\mathrm{m}-\mathrm{xylyl}) \mathrm{Rh}-\mathrm{CH}_{2} \mathrm{CH}_{3}(\mathbf{8})$ also occur as intermediates in the ethane 
$\mathrm{C}-\mathrm{H}$ bond reaction with $\mathbf{1}$ during the time period before all the dimetalloradical $\mathbf{1}$ is converted to diamagnetic species. The ethane reaction system when allowed to evolve for weeks ultimately proceeds to produce two diamagnetic complexes. The major species $(\sim 90 \%)$ is assigned by ${ }^{1} \mathrm{H}$ NMR to a diethyl complex (9) and the minor species $(\sim 10 \%)$ is an ethene bridged complex $\mathrm{Rh}(\mathrm{m}-$ xylyl)Rh- $\left(\mu-\mathrm{CH}_{2} \mathrm{CH}_{2}\right)$. Compound 9 is shown to be the diethyl derivative $\left(\mathrm{CH}_{3} \mathrm{CH}_{2}-\mathrm{Rh}(\mathrm{m}-\right.$ xylyl)Rh- $\mathrm{CH}_{2} \mathrm{CH}_{3}$ ) by comparison of the ${ }^{1} \mathrm{H}$ NMR with authentic samples prepared by directed synthesis. Formation of the ethene-bridged complex $\mathrm{Rh}(\mathrm{m}-\mathrm{xylyl}) \mathrm{Rh}-\left(\mu-\mathrm{CH}_{2} \mathrm{CH}_{2}\right)$ is confirmed by the distinctive ${ }^{1} \mathrm{H}$ NMR peaks at $-7.87 \mathrm{ppm}(\mathrm{m}),-8.38 \mathrm{ppm}(\mathrm{m})$ for the $\mu-C H_{A} H_{M} C H_{A} H_{M}$, and $5.25 \mathrm{ppm}(\mathrm{d}), 5.14 \mathrm{ppm}$ (d) for the inequivalent diasterotopic tether methylene hydrogens (OC $H_{A} H_{B}$ ) of two equivalent $-\mathrm{CH}_{A} H_{B^{-}}$groups, which are identical to the bridged complex synthesized directly from reaction of $\cdot \mathrm{Rh}\left(\mathrm{m}\right.$-xylyl) $\mathrm{Rh} \cdot$ with $\mathrm{CH}_{2}=\mathrm{CH}_{2}$ (eq. S3).

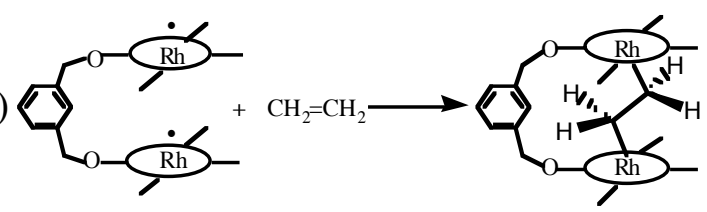

The change in concentration of $\mathbf{1}$ with time for reaction S2 is empirically fitted to a second order process ( rate $\left._{\mathrm{S} 2}=\mathrm{k}_{\mathrm{S} 2}(\mathrm{app})[\mathbf{1}][\mathrm{S}]\right)$, which yields an effective second order rate constant $\left(\mathrm{k}_{\mathrm{S} 2}(\mathrm{app})=5.3 \times 10^{-4} \mathrm{M}^{-1} \mathrm{~s}^{-1}, \mathrm{~T}=296 \mathrm{~K}\right)$. The rate constant for the methane reaction with $\mathbf{1}$ is two orders of magnitude larger than that for the ethane reaction.

The intramolecular reaction relatively quickly equilibrates $\cdot \mathrm{Rh}(\mathrm{m}$-xylyl) $\mathrm{Rh}$, $\mathrm{H}-\mathrm{Rh}\left(\mathrm{m}\right.$-xylyl) $\mathrm{Rh}-\mathrm{CH}_{2} \mathrm{CH}_{3}$ and $\mathrm{C}_{2} \mathrm{H}_{6}$ (eq. $\mathrm{S} 2$ ), which is maintained during the remaining process. Integration of the ${ }^{1} \mathrm{H}$ NMR for 1, 7 and ethane was used to evaluate the equilibrium constant for reaction $\mathrm{S} 2\left(\mathrm{~K}_{\mathrm{S} 2}(296 \mathrm{~K})=80 \pm 30 ; \Delta \mathrm{G}_{\mathrm{S} 2}{ }^{\circ}(296 \mathrm{~K})=-2.6 \pm 0.3 \mathrm{kcal} \mathrm{mol}^{-1}\right)$.

The change in concentration of $\mathbf{1}$ with the time is empirically fitted to a second order process $\left(\right.$ rate $\left._{\mathrm{S} 2}=\mathrm{k}_{\mathrm{S} 2}(\mathrm{app})[\mathbf{1}][\mathrm{S}]\right)$, which yields an effective second order rate constant $\left(\mathrm{k}_{\mathrm{s} 2}(\mathrm{app})=5.3 \times 10^{-4}\right.$ $\left.\mathrm{M}^{-1} \mathrm{~s}^{-1}, \mathrm{~T}=296 \mathrm{~K}\right)($ Figure S3). 


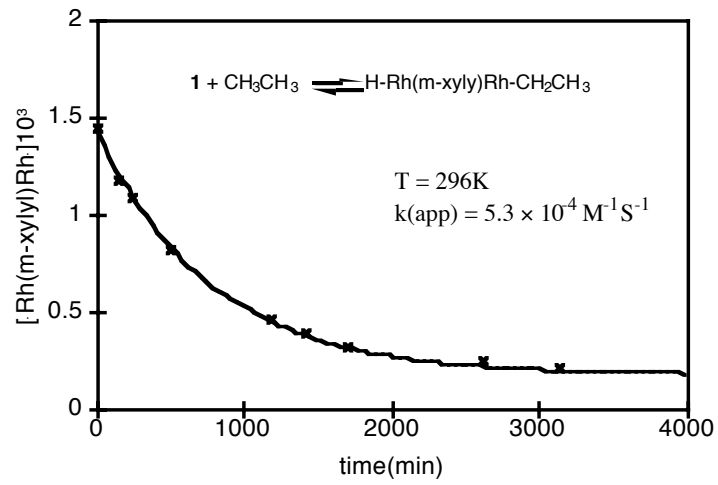

Figure S3. Change in the $[\cdot \mathrm{Rh}(\mathrm{m}-\mathrm{xylyl}) \mathrm{Rh} \cdot]$ with time for the reaction of ethane $\left(\mathrm{P}_{\mathrm{C}_{2} \mathrm{H}_{6}}=197\right.$ torr, $\left.\left[\mathrm{C}_{2} \mathrm{H}_{6}\right]=3.6 \times 10^{-2} \mathrm{M},[\cdot \mathrm{Rh}(\mathrm{m}-\mathrm{xylyl}) \mathrm{Rh} \cdot]_{\mathrm{i}}=1.50 \times 10^{-3} \mathrm{M}\right)$.

Reaction of $\cdot \mathbf{R h}(\mathbf{m}-x y l y l) R h \cdot(1)$ with Methanol: The bimetalloradical (1) reacts exclusively with the $\mathrm{C}-\mathrm{H}$ bond of $\mathrm{CH}_{3} \mathrm{OH}\left(\left[\mathrm{CH}_{3} \mathrm{OH}\right]=0.01-0.12 \mathrm{M}\right)$ and forms a dirhodium hydrido-hydroxymethyl complex (10) (eq. S4) along with two paramagnetic mono $\mathrm{Rh}^{\mathrm{II}} \cdot \mathrm{species}$ $\cdot \mathrm{Rh}(\mathrm{m}-\mathrm{xylyl}) \mathrm{Rh}-\mathrm{H}(\mathbf{3})$ and $\cdot \mathrm{Rh}(\mathrm{m}-\mathrm{xylyl}) \mathrm{Rh}-\mathrm{CH}_{2} \mathrm{OH}(\mathbf{1 1})$.

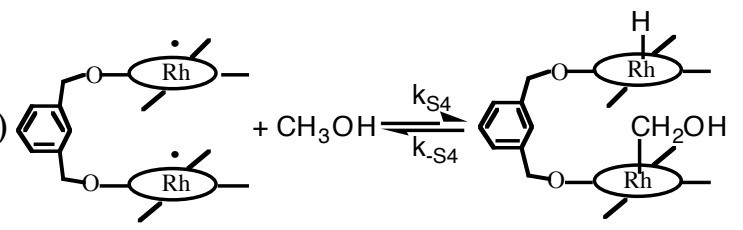

The methanol reaction system is useful for following the evolution of reaction products because ${ }^{1} \mathrm{H}$ NMR is capable of easily distinguishing among the three possible diamagnetic products $\mathrm{H}-\mathrm{Rh}(\mathrm{m}-\mathrm{xylyl}) \mathrm{Rh}-\mathrm{CH}_{2} \mathrm{OH}(\mathbf{1 0}) \mathrm{H}-\mathrm{Rh}(\mathrm{m}-\mathrm{xylyl}) \mathrm{Rh}-\mathrm{H}$ (2) and $\mathrm{HOCH}_{2}-\mathrm{Rh}(\mathrm{m}-\mathrm{xylyl}) \mathrm{Rh}-$ $\mathrm{CH}_{2} \mathrm{OH}(\mathbf{1 2})$, and also distinguishing between the three paramagnetic species $\cdot \mathrm{Rh}(\mathrm{m}$-xylyl) $\mathrm{Rh} \cdot(\mathbf{1})$, $\cdot \mathrm{Rh}(\mathrm{m}-\mathrm{xylyl}) \mathrm{Rh}-\mathrm{H}(\mathbf{3})$ and $\cdot \mathrm{Rh}(\mathrm{m}-\mathrm{xylyl}) \mathrm{Rh}-\mathrm{CH}_{2} \mathrm{OH}$ (11). The ${ }^{1} \mathrm{H}$ NMR spectra shown in Figure S4 were obtained at the time when the reacting system has just reached the point where effectively all of the paramagnetic species $(\mathbf{1}, \mathbf{3}$ and 11) have reacted to form diamagnetic species containing Rh- $\mathrm{CH}_{2} \mathrm{OH}$ and $\mathrm{Rh}-\mathrm{H}$ units. At this point of the reaction, the only observed diamagnetic species that contains a $\mathrm{Rh}-\mathrm{CH}_{2} \mathrm{OH}$ unit is the mono-hydroxymethyl derivative $\mathrm{H}-\mathrm{Rh}\left(\mathrm{m}\right.$-xylyl) $\mathrm{Rh}-\mathrm{CH}_{2} \mathrm{OH}$ (10), but the dihydride 2 is also observed as a minority diamagnetic complex. Absence of the 
dihydroxymethyl complex $\mathbf{1 2}$ at this point in the reaction is an experimental demonstration that the intramolecular use of two $\mathrm{Rh}^{\mathrm{II}}$. centers in $\mathbf{1}$ is much faster than an intermolecular pathway involving reaction of $\mathrm{Rh}^{\mathrm{II}}$. centers in two different molecules of $\mathbf{1}$. Hydrogen atom interchange between $\mathrm{Rh}^{\mathrm{II}}$. and $\mathrm{Rh}-\mathrm{H}$ centers produces the dihydride $\mathbf{3}$ and reforms $\mathbf{1}$.

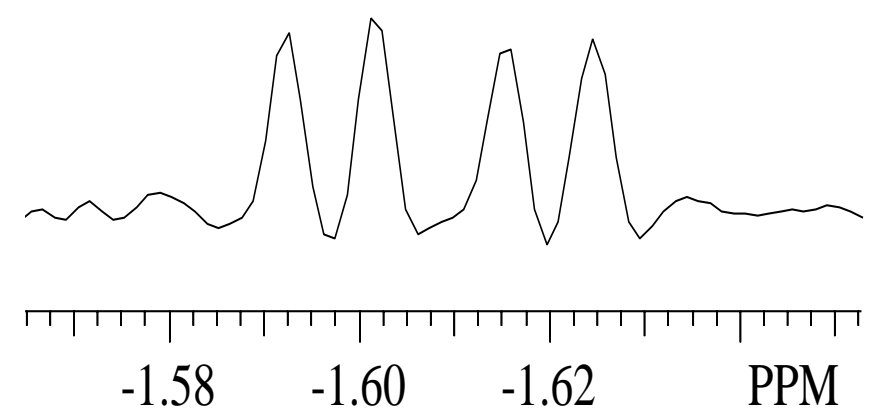

Figure S4. ${ }^{1} \mathrm{H}$ NMR of diamagnetic species present in the reaction of 1 with $\mathrm{MeOH}([\mathrm{MeOH}]=$ $0.11 \mathrm{M}, \mathrm{t}=3$ hours) at the time when all species containing rhodium(II) have reacted away in $\mathrm{C}_{6} \mathrm{D}_{6}$ at $296 \mathrm{~K}(\mathrm{a}) . \mathrm{Rh}-\mathrm{CH}_{2} \mathrm{OH}\left({ }^{3} \mathrm{~J}_{\mathrm{H}-\mathrm{H}}=8.2 \mathrm{~Hz},{ }^{2} J_{103 \mathrm{Rh}-\mathrm{H}}=3.4 \mathrm{~Hz}\right) ;(\mathrm{b}) . \mathrm{Rh}-\mathrm{CH}_{2} \mathrm{OH}\left({ }^{3} \mathrm{~J}_{\mathrm{H}-\mathrm{H}}=8.2 \mathrm{~Hz}\right) ;(\mathrm{c})$. $\mathrm{Rh}-H\left({ }^{1} J_{103 \mathrm{Rh}-\mathrm{H}}=42.8 \mathrm{~Hz}\right) ;(\mathrm{d})-\mathrm{OCH}_{2}$ - peaks

Subsequent to the near complete conversion of $\mathbf{1}$ to $\mathbf{1 0}$, the slow appearance of another diamagnetic species that contains $\mathrm{Rh}-\mathrm{CH}_{2} \mathrm{OH}$ units was observed by ${ }^{1} \mathrm{H}$. The second species formed is shown to be identical with an authentic sample of the dihydroxymethyl derivative $\mathrm{HOCH}_{2}-\mathrm{Rh}(\mathrm{m}-\mathrm{xylyl}) \mathrm{Rh}-\mathrm{CH}_{2} \mathrm{OH}(\mathbf{1 2})$ produced by directed synthesis from reaction of 2 and $\mathrm{H}_{2} \mathrm{CO}$ in benzene. Intramolecular and intermolecular processes that give reductive elimination of $\mathrm{H}_{2}$, but do not give reductive elimination of ethylene glycol permit the build up of the final dihydroxymethyl complex 12 by slow intermolecular substrate activation.

Empirically fitting the kinetics for the disappearance of $\mathbf{1}$ to the expression for a second order process approaching equilibrium gives the apparent rate constants for the reaction of $\mathrm{CH}_{3} \mathrm{OH}$ and $\mathrm{CD}_{3} \mathrm{OD}\left(\mathrm{k}_{\mathrm{S} 4}(\mathrm{app})\left(\mathrm{CH}_{3} \mathrm{OH}\right)=1.45 \times 10^{-2} \mathrm{M}^{-1} \mathrm{~s}^{-1}, \mathrm{k}_{\mathrm{S} 4}(\mathrm{app})\left(\mathrm{CD}_{3} \mathrm{OD}\right)=1.5 \times 10^{-3} \mathrm{M}^{-1} \mathrm{~s}^{-1} ; \mathrm{T}=\right.$ 
$296 \mathrm{~K})^{4}$. Temperature dependence of the apparent second order rate constants for the C-H activation reaction were used in deriving the activation parameters for the reaction of $\mathbf{1}$ with methanol $\left(\Delta \mathrm{H}_{\mathrm{S} 4}{ }^{\ddagger}(\mathrm{app})=15.6 \pm 1.0 \mathrm{kcal} \mathrm{mol}^{-1} ; \Delta \mathrm{S}_{\mathrm{S} 4}{ }^{\ddagger}(\mathrm{app})=-14 \pm 5 \mathrm{cal} \mathrm{K}^{-1} \mathrm{~mol}^{-1}\right)$.

Equilibrium among the species in reaction $\mathrm{S} 4\left(\cdot \mathrm{Rh}\left(\mathrm{m}\right.\right.$-xylyl) $\mathrm{Rh} \cdot \mathrm{H}-\mathrm{Rh}(\mathrm{m}-\mathrm{xylyl}) \mathrm{Rh}_{-} \mathrm{CH}_{2} \mathrm{OH}$ and $\mathrm{CH}_{3} \mathrm{OH}$ ) is achieved by a relatively fast bimolecular process. The equilibrium for reaction $\mathrm{S} 4$ is maintained in the reaction system as the other slow intermolecular reactions occur. Evaluation of equilibrium constant for reaction $\mathrm{S} 4$ was made by integration of its ${ }^{1} \mathrm{H}$ NMR $\left(\mathrm{K}_{\mathrm{S} 4}(296 \mathrm{~K})=(1.5 \pm\right.$ $\left.0.5) \times 10^{3} ; \Delta \mathrm{G}_{\mathrm{s} 4}{ }^{\circ}(296 \mathrm{~K})=-4.3 \pm 0.2 \mathrm{kcal} \mathrm{mol}^{-1}\right)$.

Subsequent to the near complete conversion of $\mathbf{1}$ to $\mathbf{1 0}$, the slow appearance of another diamagnetic species that contains $\mathrm{Rh}-\mathrm{CH}_{2} \mathrm{OH}$ units was observed by ${ }^{1} \mathrm{H}$ (Figure S5). The second species formed is shown to be identical with an authentic sample of the dihydroxymethyl derivative $\mathrm{HOCH}_{2}-\mathrm{Rh}\left(\mathrm{m}\right.$-xylyl) $\mathrm{Rh}-\mathrm{CH}_{2} \mathrm{OH}$ (12) produced by directed synthesis from reaction of 2 and $\mathrm{H}_{2} \mathrm{CO}$ in benzene.

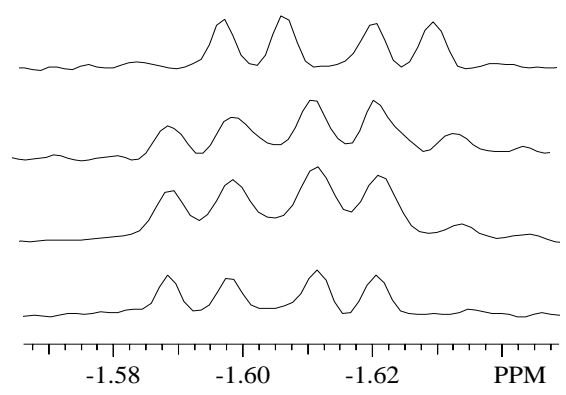

Figure S5. Time evolution of the ${ }^{1} \mathrm{H}$ NMR for the $\mathrm{Rh}-\mathrm{CH}_{2} \mathrm{OH}$ unit of the diamagnetic products of reaction 1 with $\mathrm{CH}_{3} \mathrm{OH}(0.11 \mathrm{M})$ : a) $\mathrm{t}=3$ hours $\left.\left(\delta=-1.611 \mathrm{ppm},{ }^{3} J_{\mathrm{H}-\mathrm{H}}=8.2 \mathrm{~Hz},{ }^{2} J_{103_{\mathrm{Rh}-\mathrm{H}}}=3.4 \mathrm{~Hz}\right) ; \mathrm{b}\right)$ $\mathrm{t}=11$ days; c) $\mathrm{t}=20$ days; d) authentic $\mathrm{HOCH}_{2}-\mathrm{Rh}(\mathrm{m}-\mathrm{xylyl}) \mathrm{Rh}_{-} \mathrm{CH}_{2} \mathrm{OH}\left(\delta=-1.602 \mathrm{ppm},{ }^{3} J_{\mathrm{H}-}\right.$ ${ }_{\mathrm{H}}=8.2 \mathrm{~Hz},{ }^{2} J_{103_{\mathrm{Rh}-\mathrm{H}}}=3.4 \mathrm{~Hz}$ ) (spectra were enhanced with Gussian multiplication; 10 $=\mathrm{HOCH}_{2}-\mathrm{Rh}(\mathrm{m}-$ xylyl)Rh-H; 12= $\left.\mathrm{HOCH}_{2}-\mathrm{Rh}(\mathrm{m}-\mathrm{xylyl}) \mathrm{Rh}-\mathrm{CH}_{2} \mathrm{OH}\right)$.

Empirically fitting the kinetics for the disappearance of $\mathbf{1}$ to the expression for a second order process approaching equilibrium gives the apparent rate constants for the reaction of $\mathrm{CH}_{3} \mathrm{OH}$ and 
$\mathrm{CD}_{3} \mathrm{OD}\left(\mathrm{k}_{\mathrm{S} 4}(\mathrm{app})\left(\mathrm{CH}_{3} \mathrm{OH}\right)=1.45 \times 10^{-2} \mathrm{M}^{-1} \mathrm{~s}^{-1}, \mathrm{k}_{\mathrm{S} 4}(\mathrm{app})\left(\mathrm{CD}_{3} \mathrm{OD}\right)=1.5 \times 10^{-3} \mathrm{M}^{-1} \mathrm{~s}^{-1} ; \mathrm{T}=\right.$ 296K). Temperature dependence of the apparent second order rate constants for the C-H activation reaction were used in deriving the activation parameters for the reaction of $\mathbf{1}$ with methanol $\left(\Delta \mathrm{H}_{\mathrm{s} 4}{ }^{\ddagger}(\mathrm{app})=15.6 \pm 1.0 \mathrm{kcal} \mathrm{mol}^{-1} ; \Delta \mathrm{S}_{\mathrm{s} 4}{ }^{\ddagger}(\mathrm{app})=-14 \pm 5 \mathrm{cal} \mathrm{K}^{-1} \mathrm{~mol}^{-1}\right)$ (Figure S6).

(A)
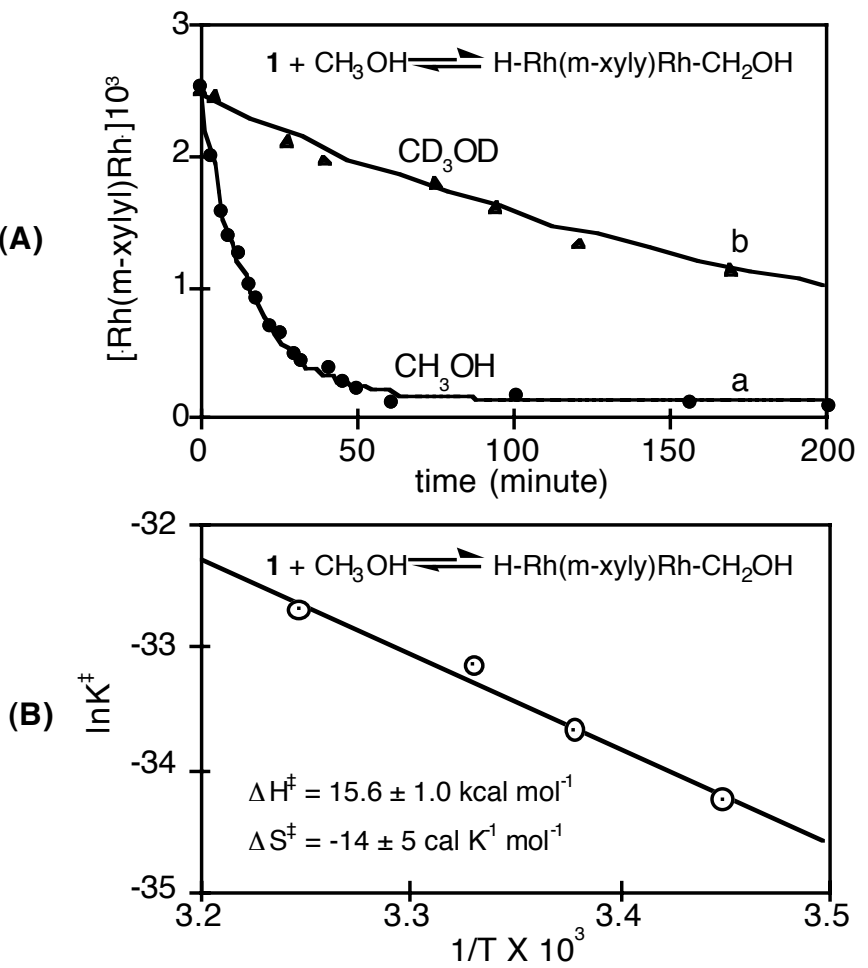

Figure S6. (A) Changes in the $[\cdot \mathrm{Rh}(\mathrm{m}-\mathrm{xy} y \mathrm{lyl}) \mathrm{Rh} \cdot]\left([\mathbf{1}]_{\mathrm{i}}=2.5 \times 10^{-3} \mathrm{M}\right)$ in $\mathrm{C}_{6} \mathrm{D}_{6}$ at $296 \mathrm{~K}$ with time in the reaction of: a) $\mathrm{CH}_{3} \mathrm{OH}(0.067 \mathrm{M}) ; \mathrm{k}\left(\mathrm{CH}_{3} \mathrm{OH}\right)(\mathrm{app})=1.45 \times 10^{-2} \mathrm{M}^{-1} \mathrm{~S}^{-1}$ and b) $\mathrm{CD}_{3} \mathrm{OD}$ $(0.050 \mathrm{M}) ; \mathrm{k}\left(\mathrm{CD}_{3} \mathrm{OD}\right)(\mathrm{app})=1.50 \times 10^{-3} \mathrm{M}^{-1} \mathrm{~S}^{-1} ;(\mathrm{B})$ Determination of the activation parameters for reaction of $\cdot \mathrm{Rh}\left(\mathrm{m}\right.$-xylyl)Rh$\cdot$ with $\mathrm{CH}_{3} \mathrm{OH}$

Reaction of $\cdot \mathbf{R h}(\mathbf{m}-x y l y l) R h \cdot(1)$ with Toluene: Toluene is observed to react with $\cdot \mathrm{Rh}\left(\mathrm{m}\right.$-xylyl)Rh - to produce $\mathrm{H}-\mathrm{Rh}(\mathrm{m}-\mathrm{xylyl}) \mathrm{Rh}-\mathrm{CH}_{2} \mathrm{C}_{6} \mathrm{H}_{5}(\mathbf{1 5})$ without any evidence for aromatic $\mathrm{C}-\mathrm{H}$ bond activation (eq. S5). The mono-hydride mono-benzyl derivative H-Rh(m-xylyl)Rh$\mathrm{CH}_{2} \mathrm{C}_{6} \mathrm{H}_{5}$ is an important example because it can be clearly distinguished from the dibenzyl derivative $\mathrm{C}_{6} \mathrm{H}_{5} \mathrm{CH}_{2}-\mathrm{Rh}\left(\mathrm{m}\right.$-xylyl) $\mathrm{Rh}-\mathrm{CH}_{2} \mathrm{C}_{6} \mathrm{H}_{5}$ in solution using only the ${ }^{1} \mathrm{H}$ NMR. Characteristic 
Rh- $H$ (d, -40.06ppm), Rh- $\mathrm{CH}_{2} \mathrm{C}_{6} \mathrm{H}_{5}\left(\mathrm{~d},-3.21 \mathrm{ppm},{ }^{2} J_{103_{\mathrm{Rh}} \mathrm{H}}=3.7 \mathrm{~Hz}\right)$ and particularly two tether methylene peaks at 4.99 and $5.04 \mathrm{ppm}$ assigned to the $-\mathrm{OCH}_{2}$ - on the inequivalent sides of $\mathbf{1 5}$ provide ${ }^{1} \mathrm{H}$ NMR identification in solution.

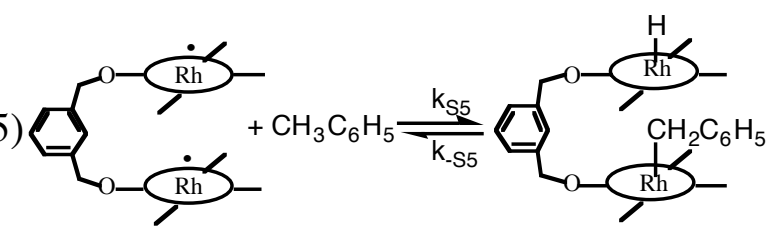

Reaction of $\mathbf{1}$ with deuterated toluene as the solvent results in formation of a diamagnetic compound that has a low resolution FAB mass spectrum showing a molecular ion of 1916.1D associated with D-Rh(m-xylyl)Rh- $\mathrm{CD}_{2} \mathrm{C}_{6} \mathrm{D}_{5}$ (1916.7D). The molecular ion peak of $\mathrm{C}_{6} \mathrm{D}_{5} \mathrm{CD}_{2}$ $\mathrm{Rh}\left(\mathrm{m}\right.$-xylyl)Rh- $\mathrm{CD}_{2} \mathrm{C}_{6} \mathrm{D}_{5}$ (2014.0D) is absent from the $\mathrm{FAB}$ mass spectrum during the early phase of the reaction. Subsequent to the near complete formation of $D-R h(m-x y l y l) R h-C_{2} C_{6} D_{5}$, the species in solution continue to evolve and ultimately a single diamagnetic species with the FAB molecular ion peak at 2013.9D is observed that is associated with $\mathrm{C}_{6} \mathrm{D}_{5} \mathrm{CD}_{2}-\mathrm{Rh}(\mathrm{m}$-xylyl)Rh$\mathrm{CD}_{2} \mathrm{C}_{6} \mathrm{D}_{5}(2014.0 \mathrm{D})$.

The formation of $\mathrm{H}-\mathrm{Rh}(\mathrm{m}-\mathrm{xylyl}) \mathrm{Rh}-\mathrm{H}$ (2) along with complex 15 was confirmed by the $\mathrm{Rh}-\mathrm{H}(-40.06 \mathrm{ppm})$ and $-\mathrm{OCH}_{2}$ - tether peaks in the ${ }^{1} \mathrm{H}$ NMR. Prior to completion of reaction S5, two paramagnetic mono-rhodium(II) species were observed and assigned to $\cdot \mathrm{Rh}(\mathrm{m}-\mathrm{xylyl}) \mathrm{Rh}-\mathrm{H}(\mathbf{3})$ and $\cdot \operatorname{Rh}(\mathrm{m}$-xylyl)Rh-benzyl (14).

The change of the $\cdot \mathrm{Rh}\left(\mathrm{m}\right.$-xylyl) Rh$\cdot$ concentration with time in the reaction of $\mathrm{CH}_{3} \mathrm{C}_{6} \mathrm{H}_{5}$ and $\mathrm{CD}_{3} \mathrm{C}_{6} \mathrm{D}_{5}$ was empirically fitted to a second order process approaching equilibrium and used in deriving an apparent second order rate constant for reaction $\mathrm{S} 5\left(\mathrm{k}_{\mathrm{S} 5}(\mathrm{app})\left(\mathrm{CH}_{3} \mathrm{C}_{6} \mathrm{H}_{5}\right)(296 \mathrm{~K})=2.3 \times\right.$ $\left.10^{-4} \mathrm{M}^{-1} \mathrm{~s}^{-1}, \mathrm{k}_{\mathrm{S} 5}(\operatorname{app})\left(\mathrm{CD}_{3} \mathrm{C}_{6} \mathrm{D}_{5}\right)(296 \mathrm{~K})=4.6 \times 10^{-5} \mathrm{M}^{-1} \mathrm{~s}^{-1}\right)$ (Figure $\left.\mathrm{S} 7\right)$. The deuterium isotopic effect for reaction S5 was obtained from the ratio of the apparent second order rate constants $\left(\mathrm{k}_{\mathrm{S} 5}(\mathrm{H}) / \mathrm{k}_{\mathrm{S} 5}(\mathrm{D})(296 \mathrm{~K})=5.0 \pm 0.7, \mathrm{~T}=296 \mathrm{~K}\right)$. The larger rate of reaction of toluene with 1 compared to the mono-metalloradical (TMP)Rh· is also illustrated in Figure S7A. 
(A)

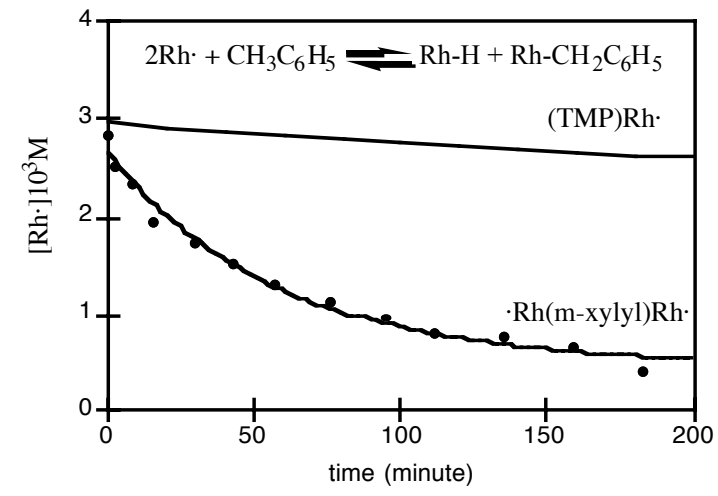

(B)

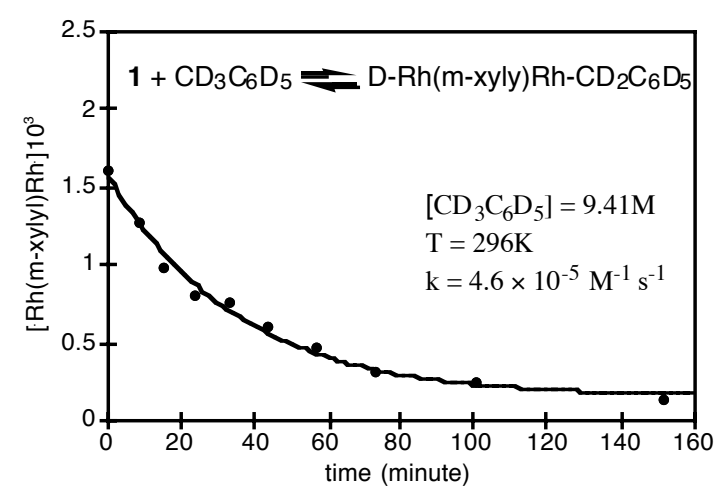

Figure S7. (A). Change in the $[\mathrm{Rh} \cdot]$ center with the time for the reaction of toluene $\left(\left[\mathrm{CH}_{3} \mathrm{C}_{6} \mathrm{H}_{5}\right]=\right.$ $0.41 \mathrm{M})$ with $(\mathrm{TMP}) \mathrm{Rh} \cdot\left(\mathrm{k}=1.04 \times 10^{-2} \mathrm{M}^{-2} \mathrm{~s}^{-1}\right)$ and $\cdot \mathrm{Rh}(\mathrm{m}-\mathrm{xylyl}) \mathrm{Rh} \cdot\left(\mathrm{k}=5.7 \times 10^{-4} \mathrm{M}^{-1} \mathrm{~s}^{-1}\right)$ in $\mathrm{C}_{6} \mathrm{D}_{6}$ at $323 \mathrm{~K}\left([(\mathrm{TMP}) \mathrm{Rh} \cdot]_{\mathrm{i}}=2.96 \times 10^{-3} \mathrm{M} ;[\cdot \mathrm{Rh}(\mathrm{m}-\mathrm{xylyl}) \mathrm{Rh} \cdot]_{\mathrm{i}}=1.4 \times 10^{-3} \mathrm{M}\right) ;(\mathrm{B})$. Change in the $[\cdot \operatorname{Rh}(\mathrm{m}-\mathrm{xylyl}) \mathrm{Rh} \cdot]$ center with the time for the reaction of $\cdot \mathrm{Rh}(\mathrm{m}-\mathrm{xylyl}) \mathrm{Rh} \cdot$ with pure toluene- $\mathrm{d}_{8}$ $\left([\cdot \operatorname{Rh}(\mathrm{m}-x y l y l) \mathrm{Rh} \cdot]_{\mathrm{i}}=1.54 \times 10^{-3} \mathrm{M}\right)$.

Temperature dependence of the apparent second order rate constant $\left(\mathrm{k}_{5}(\mathrm{app})\right)$ yields activation parameters for the reaction of 1 with toluene $\left(\Delta \mathrm{H}_{\mathrm{S} 5}{ }^{*}(\operatorname{app})\left(\mathrm{C}_{7} \mathrm{H}_{8}\right)=5.6 \pm 0.5 \mathrm{kcal} \mathrm{mol}^{-1}\right.$; $\left.\Delta \mathrm{S}_{\mathrm{S} 5}{ }^{\ddagger}(\mathrm{app})\left(\mathrm{C}_{7} \mathrm{H}_{8}\right)=-56 \pm 5 \mathrm{cal} \mathrm{K}^{-1} \mathrm{~mol}^{-1}\right)$ and toluene- $\mathrm{d}_{8}\left(\Delta \mathrm{H}_{\mathrm{S} 5}{ }^{\ddagger}(\mathrm{app})\left(\mathrm{C}_{7} \mathrm{D}_{8}\right)=7.6 \pm 1.2 \mathrm{kcal} \mathrm{mol}^{-}\right.$ $\left.{ }^{1} ; \Delta \mathrm{S}_{\mathrm{S} 5}{ }^{\ddagger}(\operatorname{app})\left(\mathrm{C}_{7} \mathrm{D}_{8}\right)=-53 \pm 8 \mathrm{cal} \mathrm{K}^{-1} \mathrm{~mol}^{-1}\right)($ Figure S8).

Relatively fast reaction of $\mathbf{1}$ with toluene produces an equilibrium distribution of $\cdot \mathrm{Rh}(\mathrm{m}-$ xylyl)Rh', $\mathrm{H}-\mathrm{Rh}(\mathrm{m}-\mathrm{xylyl}) \mathrm{Rh}-\mathrm{CH}_{2} \mathrm{C}_{6} \mathrm{H}_{5}$ and $\mathrm{CH}_{3} \mathrm{C}_{6} \mathrm{H}_{5}$ that is maintained as the process moves toward completion. The equilibrium constant and free energy change in reaction S5 were evaluated at $296 \mathrm{~K}\left(\mathrm{~K}_{\mathrm{S} 5}=5.0 \pm 0.5 ; \Delta \mathrm{G}_{\mathrm{S} 5}^{\circ}=-1.0 \pm 0.1 \mathrm{kcal} \mathrm{mol}^{-1}\right)$. 


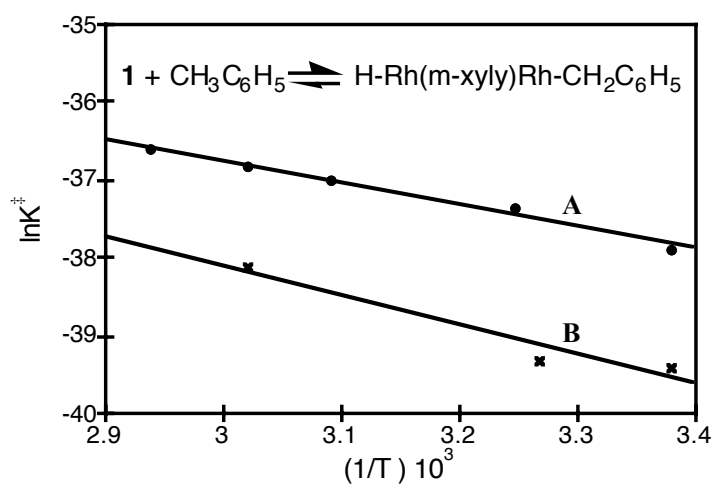

Figure S8. Determination of the activation parameters for reactions of toluene with $\cdot \mathrm{Rh}(\mathrm{m}-$ xylyl)Rh· in $\mathrm{C}_{6} \mathrm{D}_{6}$ : A) $\mathrm{C}_{6} \mathrm{H}_{5} \mathrm{CH}_{3}$; B) $\mathrm{C}_{6} \mathrm{D}_{5} \mathrm{CD}_{3}$

\section{Reference:}

1. Zhang, X.-X.; Wayland, B. B. Inorg. Chem. 2000, 39, 5318.

2. Wayland, B. B.; Ba, S.; Sherry, A. E. J. Am. Chem. Soc. 1991, 113, 5305.

3. Zhang, X.-X.; Wayland, B. B. J. Am. Chem. Soc. 1994, 116, 7897.

4. Cui, W.; Zhang, X. P.; Wayland, B. B. J. Am. Chem.. Soc. 2003, 125, 4994 